\title{
Cyclic Di-GMP: Second Messenger Extraordinaire
}

3

4

5

6

Urs Jenal*, Alberto Reinders ${ }^{ \pm}$, and Christian Lori ${ }^{ \pm}$

7

Biozentrum of the University of Basel

Klingelbergstrasse 50/70, 4054 Basel, Switzerland

*for correspondence: urs.jenal@unibas.ch

${ }^{ \pm}$equal contribution 


\section{Abstract}

Cyclic dinucleotides are highly versatile signaling molecules in both prokaryotes and eukaryotes involved in the control of various important biological processes. The best-studied example is bis-(3'-5')-cyclic dimeric GMP (c-di-GMP). Known since the late 1980's it is now recognized as near-ubiquitous second messenger in bacteria that coordinates diverse aspects of bacterial growth and behavior including motility, virulence, biofilm formation or cell cycle progression. In this Review, we discuss important new insights into the molecular principles of c-di-GMP synthesis and degradation, and its function in cellular control and give a short overview on the signaling versatility of other CDNs including c-di-AMP, cAMP-GMP and cGAMP.

\section{Introduction}

The roles of the prototypical second messengers cAMP and cGMP have been studied for over 50 years, whereas recognition of their larger relatives, the cyclic dinucleotides (CDNs), lagged behind. The first CDN was discovered in 1987, when Moshe Benziman reported on "an unusual cyclic nucleotide activator" that was able to stimulate cellulose synthase from Acinetobacter xylinum and identified this compound as bis-(3'-5')-cyclic diguanylic acid (cdi-GMP) ${ }^{1}$. More than 20 years later, c-di-AMP was discovered as a factor involved in DNA repair in Bacillus subtilis ${ }^{2}$. Moreover, different versions of c-GMP-AMP (cGAMP) were first discovered in bacteria ${ }^{3}$ and later in mammalian cells ${ }^{4}$ and were shown to have prominent roles in bacterial virulence and the innate immune response. Despite of their chemical similarities, different CDNs seem to have distinct evolutionary origins with enzymes involved in their synthesis and breakdown being structurally unrelated ${ }^{2,5,6}$. The idea that different CDNs evolved in parallel emphasizes the potency and versatility of this macrocyclic ring with two purine moieties as key carrier of cellular information.

The discovery of CDNs has provided novel entry points into studying important biological processes and cell behavior, including how bacteria coordinate their own growth and replication cycle, how they adapt to surfaces by forming multicellular consortia called biofilms, or how pathogenic bacteria control their virulence and persistence. This was possible by first identifying the enzymes involved in CDN synthesis and degradation, followed by the characterization of specific effectors and target molecules. The CDN field is now rapidly expanding into different directions exploring signaling aspects at the atomic, molecular and cellular lev- 
els. In the past years, we have learned that CDNs are widespread and immensely versatile signaling molecules that regulate cellular processes at multiple levels of control and that are well integrated with other global regulatory pathways in bacteria like phosphorylation networks $^{7}$, quorum sensing ${ }^{8}$ or with other small signaling molecules like cGMP, cAMP or $\mathrm{ppGpp}^{9-11}$. In this Review, we discuss some of the recent advances in CDN biology without the claim to be comprehensive and with a primary focus on aspects of c-di-GMP signaling. Cdi-GMP is not only the most widespread CDN in bacteria but so far is also the most intensely studied and best understood member of this family of second messengers. We first summarize properties of the core components of c-di-GMP signaling, enzymes involved in its synthesis and breakdown as well as effector proteins that convert dynamic changes of c-di-GMP concentrations into specific cellular responses. We then highlight recent progress in understanding the most prominent cellular processes regulated by c-di-GMP. While we draw some parallels between c-di-GMP and other CDNs, c-di-AMP signaling in bacteria and the role of cGAMP in the mammalian innate immune response will not be discussed in detail. Instead we refer readers to some excellent and comprehensive recent reviews on CDNs and their prominent role in bacterial physiology $2,4,12-16$.

\section{Makers and breakers}

The c-di-GMP monomer shows 2-fold symmetry with two GMP moieties fused by a 5'3' macrocyclic ring (Fig. 1a). High-resolution structures of c-di-GMP, in solution or bound to protein, indicated that the ligand exists either as elongated monomer or as condensed intercalated dimer ${ }^{3,17}$. At physiological concentrations c-di-GMP is a monomer in solution ${ }^{4,18}$ arguing that intercalated dimers form by successive binding of two molecules to specific effector proteins. Cellular levels of c-di-GMP are regulated in response to environmental and internal cues. This is achieved through the activity of two antagonistic enzyme families, diguanylate cyclases (DGCs) and c-di-GMP specific phosphodiesterases (PDEs) (Figure 1a) with equivalent enzyme activities being responsible for c-di-AMP metabolism (Box 2). DGCs and PDEs are found in members of all major bacterial phyla, representing two of the largest known families of signaling proteins in the bacterial kingdom ${ }^{2,5,6,12}$. The synthesis of c-di-GMP is catalyzed by DGCs through the cooperative action of two catalytic GGDEF domains that arrange in an antiparallel fashion with one GTP molecule bound by each protomer. Pioneering structural and mechanistic studies with PleD, a DGC from Caulobacter crescentus, proposed modes of substrate binding, catalytic mechanism, enzyme activation and product inhibition 
for this class of enzymes ${ }^{5,7,19-21}$. A metal-catalyzed mechanism was proposed, whereby two GTP molecules are positioned in an antiparallel manner to enable the formation of two intermolecular phosphodiester bonds ${ }^{8,22}$ (Figure 1b). The requirement for dimerization conveys a simple mechanism to control DGC activity by using an accessory domain that forms homodimers in a signal-dependent manner. In the case of PleD or its Pseudomonas aeruginosa homolog WspR this is facilitated by an N-terminal receiver domain, which dimerizes upon phosphorylation $^{5,9-11,19,23}$ (Figure 1b). An alternative mechanism for the activation of DGCs was proposed recently for DgcZ from Escherichia coli, an enzyme with a catalytic GGDEF domain fused to an N-terminal zinc-binding (CZB) domain. DgcZ is a constitutive dimer with its activity being allosterically regulated by the CZB domain ${ }^{22}$ (Figure 1c). When zinc is present, the GGDEF domains of DgcZ, although facing each other, are not positioned in a catalytically competent conformation. DgcZ activation in the absence of zinc may occur via repositioning of the GGDEF domains to enable the productive encounter of bound substrates molecules (Figure 1b).

The arrangement of the catalytic GGDEF domains was also implicated in feedback inhibition. Many of these enzymes are subject to non-competitive product inhibition by binding of c-di-GMP to the allosteric I-site on the surface of the GGDEF domain ${ }^{5,21}$. In PleD or WspR, an intercalated c-di-GMP dimer binds to this primary site and to a secondary binding site thereby immobilizing the GGDEF domains in a non-productive state ${ }^{19,23}$ (Figure 1b). Product inhibition of DGCs may establish precise cellular threshold concentrations of c-di-GMP or contribute to the reduction of stochastic perturbations and increased stability of c-di-GMP networks by maintaining c-di-GMP levels in defined concentration windows ${ }^{21}$. While a functional connection between the I-site and product inhibition is clearly established, c-di-GMP binding to some GGDEF domains may also serve other purposes like protein-protein interac$\operatorname{tion}^{24}$ (see chapter on receptors, below).

Structurally and mechanistically distinct c-di-GMP specific PDEs have been described that are based on EAL and HD-GYP domains, respectively. EAL-type PDEs hydrolyze c-diGMP in the presence of $\mathrm{Mg}^{2+}$ or $\mathrm{Mn}^{2+}$ to yield the linear $\mathrm{pGpG}$ dinucleotide ${ }^{25}$. EAL domaincontaining proteins are active as dimers ${ }^{26,27}$. But in contrast to DGCs, where the fusion of two GTP molecules requires a dimer arrangement of the enzyme, the necessity of this quarternary arrangement for PDE catalysis is not intuitive. Recent structural studies implied a regulatory role for EAL dimerization. Based on distinct structural arrangements of EAL dimers, a clamshell-like opening and closing of the EAL dimer was proposed to regulate PDE activity ${ }^{27,28}$. The evolutionary conserved dimerization interface is formed by two helices, $\alpha 5$ and $\alpha 6$, with 
$\alpha 5$ directly connecting via the $\beta 5-\alpha 5$ loop (loop 6) to two central Asp residues that coordinate the metal ions in the active site ${ }^{26,27,29}$ (Figure 1d). Structural and biophysical studies revealed that the $\alpha 5$-loop6 region undergoes substantial rearrangements during the clam-like movements of the EAL dimer, indicating that this part of the protein may play a hinge-joint-like role to couple EAL conformation to catalytic activity via the positioning of metal ions in the active site ${ }^{27,28}$. Consistent with this, accessory domains known to control PDE activity directly communicate with the EAL dimerization region ${ }^{28}$. The observation that substrate binding can induce EAL dimerization and also determines the conformation of the $\alpha 5$-loop6 region proposed a bidirectional allosteric communication between EAL domains and regulatory domains with the $\alpha 5$-loop6 region serving as central communication platform ${ }^{27-29}$. Interestingly, EAL domain-containing proteins that have adopted roles as c-di-GMP effectors seem to exploit similar c-di-GMP-mediated dimerization and $\alpha 5$-loop6 remodeling to regulate cellular processes $^{30}$ (see below).

A second, unrelated family of c-di-GMP-specific phosphodiesterase harbors conserved HD-GYP domains ${ }^{31}$. Recently, the first structure of an active HD-GYP PDE was solved implicating a novel trinuclear iron-binding site in catalysis ${ }^{32}$. While EAL-based enzymes convert c-di-GMP into the linear product pGpG, HD-GYP hydrolyzes c-di-GMP in a one-step reaction to yield two molecules of GMP ${ }^{32}$. Thus, for bacteria that lack HD-GYP-domain proteins it remained unclear how pGpG is further catabolized into GMP. This puzzle was solved recently by demonstrating that the oligoribonuclease Orn, a ribonuclease hydrolyzing two- to five-nucleotide-long RNAs, is the primary enzyme capable of removing $\mathrm{pGpG}^{33,34}$.

Despite detailed knowledge on structure and function of DGCs and PDEs, it has remained challenging to assign physiological roles to individual enzymes under laboratory conditions. Genetic studies often fail to disclose clear phenotypes. Since only few specific input signals have been identified for these enzymes so far, this may be due to the limited physiological conditions that are assayed in the laboratory. Evidence for this was provided by a recent study of PDEs in E. coli. Despite of a total of 13 PDEs being encoded in the genome of this organism, only PdeH is able to reduce c-di-GMP levels and license motility in growing $E$. coli cells $\mathrm{s}^{35,36}$ (see below). The observations that most PDEs are readily expressed and that a large fraction of these enzymes can be genetically activated to substitute for PdeH in motility control implied that most of these enzymes simply lack the appropriate stimuli under laboratory conditions ${ }^{37}$. DGCs and PDEs also engage in downstream signaling through direct interactions with their target molecules thereby providing a platform for "spatially localized" control of cellular processes ${ }^{24,38,39}$. Within such supra-molecular complexes, these proteins not 
only regulate the synthesis and degradation of c-di-GMP, but can also act as "c-di-GMP sensors" to control neighboring partner proteins ${ }^{39}$.

\section{C-di-GMP receptors}

While "makers and breakers" explain how c-di-GMP levels are controlled in time and space, c-di-GMP pathways ultimately hinge on the respective effectors that bind c-di-GMP and on their downstream targets, cellular components that are regulated by specific c-di-GMP effectors. Given the global influence of c-di-GMP on bacterial cell physiology and given the sheer abundance of DGCs and PDEs in some bacteria, it must be assumed that a large number of such effectors and cellular targets exist. Several families of effector proteins and RNAs have been identified and are now well characterized structurally and functionally ${ }^{40}$. This includes mRNA riboswitches ${ }^{41}$, transcription regulators ${ }^{42}$, proteins containing PilZ domains, a small prototypical c-di-GMP binding unit ${ }^{36,43,44}$ and proteins harboring degenerate GGDEF and EAL domains ${ }^{45}$. With one of these examples the field has recently come full circle. The discovery of c-di-GMP goes back to the observation that c-di-GMP activates the membranebound $\mathrm{Bcs} \mathrm{AB}$ cellulose synthase complex in Gluconacetobacter xylinus thereby boosting the production of this exopolysaccharide matrix component ${ }^{1}$. The availability of the structure of the BcsAB complex now revealed an elegant mechanism, whereby c-di-GMP binding to the C-terminal PilZ domain of BcsA releases autoinhibition of the glycosyltransferase activity to activate the complex ${ }^{46}$ (Figure 1e). This example illustrates how c-di-GMP effectors such as PilZ or the newly discovered YajQ protein family ${ }^{47}$ can act as versatile adaptors that link c-diGMP signal input to the activity of enzymes complexes or transcription factors.

The discovery that c-di-GMP binds to a range of transcription factors such as members of the response regulator or CRP/FNR families in a way that was not predictable from protein sequence, argued for a more versatile nature of effector-ligand interactions ${ }^{48-50}$. This is supported by the identification and characterization of a range of novel c-di-GMP effectors, an endeavor that was greatly aided by the introduction of innovative high-throughput methods and biochemical techniques (Box 1). One of the most exciting recent discoveries is the emergence of ATPases as molecular targets of c-di-GMP. The first example is FleQ, a bacterial enhancer-binding protein from $P$. aeruginosa. While members of this family of transcription factors are normally activated by phosphorylation, FleQ activity is controlled by c-di-GMP ${ }^{51}$. Structural studies revealed that the second messenger interacts with the AAA+ ATPase domain of FleQ at a site distinct from the ATP binding pocket. Binding of c-di-GMP obstructs 
FleQ ATPase activity, thereby altering its quaternary structure and transcriptional activity ${ }^{50}$. Similarly, c-di-GMP specifically binds to MshE, an AAA ${ }^{+}$ATPase involved in the assembly of mannose sensitive hemagglutinin (MSHA) pili in Vibrio cholera $e^{52,53}$. The observation that HxrA, a MshE homolog and type 2 secretion (T2S) ATPase from P. aeruginosa also specifically binds c-di-GMP opened up the exciting possibility that this general protein secretion pathway that employs a pilus-like extrusion mechanism might also be controlled directly by c-di-GMP ${ }^{52}$. The idea that c-di-GMP might have taken a more global control over bacterial protein secretion is reinforced by some recent observations indicating that this second messenger also controls Type $6(\mathrm{~T} 6 \mathrm{~S})^{54}$ as well as Type 3 secretion (T3S $)^{55}$. While the exact role of c-di-GMP in T6S is yet unclear, its influence on T3S seems to be direct and again mediated via a central ATPase. It was shown that the flagellar export ATPase FliI from a range of distantly related bacteria specifically bind c-di-GMP ${ }^{55}$. Binding of c-di-GMP to FliI and to its homolog HrcN from the virulence related T3SS inhibits ATPase activity arguing that it directly interferes with flagellar export and T3S. The authors of this study proposed that the c-diGMP binding arrangement might be widely conserved among the rotary export ATPases, making the second messenger central to the function of many of these secretion proteins. It will be interesting to compare the c-di-GMP binding mode of the individual members of this family once structural information is available. Finally, sensor histidine kinases, the central components of phosphorylation pathways in bacteria, have also been identified as c-di-GMP targets. The histidine kinase CckA from C. crescentus was shown to bind c-di-GMP via its catalytic and ATPase domain, thereby shifting the kinase/phosphatase balance of this bifunctional enzyme ${ }^{7}$ (see below). The discovery that ATPases serve as regulatory hubs for c-diGMP may reflect on the global role of c-di-GMP in monitoring bacterial cell physiology. ATPases often function as central regulatory switches governing key cellular processes. Apparently, c-di-GMP leverages part of its global influence by seizing control over these essential cellular players.

\section{Physiological roles of c-di-GMP}

\section{Development and morphogenesis}

Several bacteria make use of c-di-GMP to control morphogenesis and developmental transitions. This includes Caulobacter crescentus, an aqueous organism with an inherently asymmetric life cycle. C. crescentus produced two specialized progeny cells during each division cycle, a motile swarmer (SW) and a sessile stalked cell (ST). Predivisional cells of $C$. 
crescentus are highly polarized with a stalk and adhesive holdfast exposed at one cell pole and a flagellum, pili and a chemotaxis apparatus assembled at the opposite pole. While the surface attached ST progeny re-initiates chromosome replication (S-phase) and cell division (G2-phase) immediately following division, the newborn SW cell is motile but blocks replication throughout an extended period (G1-phase). Replication and division resume when the SW cell differentiates into a ST cell, a process during which it ejects its flagellum, retracts its pili and replaces them with a holdfast and a stalk ${ }^{56}$ (Figure 2). Recent studies identified c-diGMP as a major driver of C. crescentus pole morphogenesis and cell cycle control ${ }^{7,57,58}$. Mutants unable to synthesize c-di-GMP lost all polar appendages and showed striking cell morphology aberrations ${ }^{58}$. Levels of c-di-GMP oscillate during the $C$. crescentus cell cycle with trough values in the motile SW, a peak during the swarmer-to-stalked cell transition and intermediate concentrations during division ${ }^{58,59}$ (Figure 2). The increase in c-di-GMP concentration during the SW-to-ST transition is produced primarily by PleD, a DGC that is activated when cells enter S-phase ${ }^{58,60}$. PleD activity is confined to the ST cell by two antagonistic histidine kinases, PleC and DivJ, which position to opposite poles of dividing cells and differentially segregate into SW and ST progenies (Figure 2). While PleC acts as phosphatase keeping $\mathrm{PleD}$ P levels low in SW cells, DivJ acts as kinase to impel PleD phosphorylation in ST cells $^{60}$. Counteracting PDEs are thought to keep c-di-GMP levels low in the motile SW cell. One of these, PdeA, localizes to the flagellated pole before division and later partitions into the newborn SW cell where it authorizes motility by keeping c-di-GMP levels low. PdeA is removed by specific proteolysis during the SW-to-ST transition coincident with PleD activation, thereby contributing to the sharp upsurge of c-di-GMP at this stage of the cell cycle ${ }^{57}$.

But how does c-di-GMP oscillation instigate the exact timing of $C$. crescentus cell cycle events? The TipF-TipN pathway regulating flagellar polarity illustrates such an example. Upon binding of c-di-GMP, TipF localizes to the pole opposite of the stalk where it connects with its polar receptor, the birth scar protein $\mathrm{TipN}^{61}$. TipF then recruits flagellar proteins to this subcellular site to initiate flagellar assembly in the predivisional cell. TipF is stable when bound to c-di-GMP but is rapidly degraded when c-di-GMP levels drop in the SW cell. Removal of TipF was proposed to reset the flagellar polarization state and to avoid mispositioning of the flagellar motor at the incipient stalked cell pole ${ }^{61}$ (Figure 2). Recent studies also linked c-di-GMP oscillations to the G1-S cell cycle transition and chromosome replication control. The transcription factor CtrA acts as inhibitor of replication initiation in C. crescentus. CtrA is phosphorylated and active in swarmer cells (G1) where it binds to the origin of replication (Cori) to block replication initiation ${ }^{56}$. During differentiation into ST cells, CtrA is 
inactivated to license replication start. CtrA activity is controlled by the bifunctional cell cycle histidine kinase CckA, which phosphorylates CtrA through the phosphotransfer protein ChpT. CckA exhibits kinase activity in the SW cell but adopts strong phosphatase activity during the G1-S transition, thereby reversing the phosphate flux through the CckA-ChpT-CtrA cascade and inactivating CtrA. Concurrent with its dephosphorylation, CtrA is degraded by the ClpXP protease $^{56}$. Both dephosphorylation and degradation of CtrA are controlled by the c-di-GMP upshift during G1-S. While degradation is mediated by the ClpXP protease adaptor PopA, which binds to c-di-GMP and delivers CtrA to the protease ${ }^{45,62,63}, \mathrm{CtrA}$ inactivation results from c-di-GMP directly interfering with the CckA kinase-phosphatase switch. Biochemical and structural studies demonstrated that c-di-GMP binds to CA domain of CckA, thereby inhibiting its default kinase activity and stimulating phosphatase activity (Figure 2$)^{7}$. In addition to adopting a cyclin-like role to drive G1-S, c-di-GMP imposes spatial control on CckA during division to install asymmetric replication of future daughter cells. In predivisional cells CckA positions to opposite cell poles, adopting kinase and phosphatase activity at the flagellated and stalked pole, respectively. This leads to a gradient of $\mathrm{CtrA} \sim \mathrm{P}$ in the cell and to asymmetric replication initiation with the Cori at the ST pole being activated before cell division is completed, while the Cori at the flagellated pole remains inactive ${ }^{64,65}$. The unequal distribution of c-di-GMP was proposed to control differential activity of CckA at opposite poles. While the bulk of dividing cells experiences high levels of c-di-GMP, a microenvironment with low levels of c-di-GMP was proposed to promote CckA kinase activity at the flagellated pole $^{7}$ (Figure 2). The authors of this study proposed that CckA sequestration to the flagellated pole could shield the protein from the cellular pool of c-di-GMP. How such a low c-di-GMP microenvironment is organized and which PDEs are involved in this spatial control remains to be shown.

Asymmetric distribution of c-di-GMP during cell division was also observed in other bacteria arguing that this might represent a general principle controlling cell behavior and/or reproduction $^{59}$. For example, during the $P$. aeruginosa cell cycle, c-di-GMP levels drop during a short period after cell division in the daughter cell that inherits the polar flagellum. This pattern is caused by the asymmetric distribution of Pch, a PDE that during division localizes to the chemotaxis machinery at the flagellated cell pole ${ }^{66}$. Akin to the G1 period of the Caulobacter cell cycle, reduction of c-di-GMP at this stage of the $P$. aeruginosa division cycle may promote diversity in the swimming behavior, which in turn could help to adapt to new environments. 
Streptomycetes undergo a complex life cycle with two distinct filamentous cell forms. Germinating spores develop into vegetative hyphae, which grow into the substrate to scavenge nutrients. Upon nutrient depletion aerial hyphae are formed, which eventually differentiate into long chains of spores ${ }^{67}$. Recently, c-di-GMP was found to have a key role in the transition from vegetative mycelial growth to the formation of a reproductive aerial mycelium ${ }^{42}$. Deletion of genes encoding proteins involved in c-di-GMP metabolism had a notable effect on colony morphology and development ${ }^{67}$. Moreover, increasing internal levels of c-di-GMP blocked development, while depleting c-di-GMP caused premature spore production bypassing the formation of aerial hyphae ${ }^{42}$. Premature sporulation is also observed in mutants lacking BldD, the master regulator of Streptomyces development that represses a global regulon of $\sim 170$ sporulation genes ${ }^{67}$. Recently, a direct connection between these two key components of developmental control was identified when BldD was shown to be a c-di-GMP effector protein that represses its target genes in a manner that depends on its binding to c-di-GMP ${ }^{42}$. A drop in cytoplasmic c-di-GMP levels, which causes the BldD dimer to fall apart and dissociate from the DNA, may then trigger BldD inactivation and sporulation. Other examples illustrating the broad impact of c-di-GMP on development and morphogenesis in bacteria include Myxococcus xanthus ${ }^{68}$, Bdellovibrio bacteriovorus ${ }^{69}$ or cyanobacteria $^{70}$.

\section{Motile-sessile transition and biofilm formation.}

Controlling the motile-sessile transition of bacteria is a universal feature of c-di-GMP. Generally, low levels of c-di-GMP are associated with motility of individual cells, while increased c-di-GMP concentrations direct bacteria into surface attached communities and biofilms. But rather than being a simple on/off switch, complex regulatory steps seem to be involved in a multi-stage process leading to surface colonization ${ }^{71}$. In line with motility being a primary target of c-di-GMP, building and operating the bacterial flagellar motor is highly regulated. This includes regulation of flagellar gene expression ${ }^{49,51}$, motor assembly ${ }^{55,61}$ or motor function $^{36,72}$. While controlling flagellar gene expression is likely to be part of a long-term adaptation strategy, tuning motor activity might be important for rapid decisions during bacterial surface encounter. For example, in E. coli and Salmonella enterica increased c-di-GMP levels result in flagellar obstruction by the c-di-GMP effector protein YcgR, which in its c-di-GMPbound form interacts with the flagellar rotor/stator interface ${ }^{36,73}$ (Figure 3a). To block YcgR activity and to authorize swimming these bacteria co-express the PDE PdeH together with their flagellar genes. A similar mechanisms was proposed to tune motility in Bacillus subtilis, where PdeH controls motility by preventing flagellar obstruction by the YcgR homolog 
$\operatorname{DgrA}^{74}$. YcgR has high ligand affinity arguing that the flagellar motor may respond to small spikes of c-di-GMP that are required to initiate surface attachment. Consecutive steps of surface colonization may involve incremental steps of c-di-GMP increase and the sequential activation of distinct cellular processes. This could be accomplished by a successive intervention of DGCs harboring distinct levels of feedback inhibition ${ }^{21}$ and by the activation of c-diGMP receptors with gradually reduced affinities ${ }^{75}$. For example, in P. aeruginosa different DGCs, PDEs and receptor proteins are required at discrete stages of biofilm formation ${ }^{71}$.

Upon surface contact, bacteria rapidly change their program, expose adhesins, activate surface motility organelles and produce an extracellular matrix to protect the developing microcolonies. This adaptation is coordinated by c-di-GMP at the transcriptional (e.g. ${ }^{76}$ ), translational (e.g. ${ }^{77}$ ) and posttranslational level (e.g. ${ }^{78}$ ). For example, c-di-GMP regulates Type IV pili (T4P), the prototypical surface adherence and motility organelles, in various bacteria including M. xanthus ${ }^{79}$, . cholera $^{53}, P$. aeruginosa ${ }^{80}$, C. $_{\text {crescentus }}{ }^{58}$ or Clostridium difficile ${ }^{81}$ (Figure 4). Likewise, in E. coli the production of the two principle biofilm matrix components, curli fibers and cellulose, is regulated by c-di-GMP ${ }^{82}$. During the motile-sessile switch, c-di-GMP levels increase as a result of $\sigma^{\mathrm{S}}$-induced expression of DgcE and other DGCs and the consecutive downregulation of the PDE PdeH, which acts as gatekeeper for motility and is part of the large flagellar regulon ${ }^{36,83}$ (formerly: $\mathrm{YegE}$ and $\mathrm{YhjH}^{35}$ ). Increased global c-diGMP levels then set in motion a local control module consisting of DgcM and PdeR, a DGC/PDE pair that directly interacts with and stimulates the transcription factor MlrA, which in turn activates the expression of the central curli regulator CsgD. Interestingly, the role of PdeR and DgcM is not primarily a catalytic one but rather to sense the global increase in c-diGMP and in response serve as co-activators for $\mathrm{MlrA}^{39}$. CsgD then mediates transcription of curli genes and at the same time induces the expression of $\mathrm{DgcC}$, the primary DGC to allosterically activate cellulose production via the cellulose synthase complex (Figures 1e, 3b) ${ }^{46}$. This is a prime example of how different levels of the c-di-GMP network are interconnected to generate highly flexible and expanding responses, which in this case enable for differential tuning of individual matrix components. An alternative exopolysaccharide, poly-beta-1,6-Nacetyl-glucosamine (PGA) can promote E. coli surface adherence and biofilm formation. PGA biogenesis and secretion requires the Pga complex (PgaA-D) and its allosteric activation by c-di-GMP. Both the pgaABCD operon and two DGCs, DgcT and DgcZ, are controlled by Csr, a global regulatory system that mediates $E$. coli virulence and biofilm formation ${ }^{84}$. Recent findings indicated that c-di-GMP activates the Pga machinery by binding directly to both 
PgaC and PgaD, the two inner membrane components of the Pga complex to stimulate their glycosyltransferase activity $^{78}$ (Figure $3 \mathrm{c}$ ).

While the processes driving biofilm formation are relatively well understood, mechanisms underlying biofilm dispersal have remained understudied. Given the prominent role of c-di-GMP in biofilm formation, careful control of the second messenger must also be linked to active biofilm dispersal ${ }^{85}$. Such an escape mechanism was identified in Pseudomonas fluorescens, where the LapA surface protein mediates surface adhesion and stabilization of biofilms ${ }^{86}$. At high c-di-GMP levels, c-di-GMP binds to LapD to help sequester the LapG protease in the periplasm. When c-di-GMP levels drop upon induction of the PDE RapA, LapD is inactivated thereby releasing the protease to cleave the LapA adhesin and to weaken the biofilm (Figure 3d).

As biofilms contribute to acute and chronic infections, it is not surprising that the c-diGMP network is under selective pressure in human patients. Slow growing, autoaggregative $P$. aeruginosa isolates from airways of patients with cystic fibrosis were shown to harbor mutations leading to strong activation of some of the major DGCs ${ }^{87,88}$. The observation that such variants effectively persisted in animal models and in the presence of sub-inhibitory concentration of antibiotics, despite of reduced growth rates in vitro, indicated that they may have an important role in persistence during antimicrobial chemotherapy ${ }^{87}$.

\section{Role of c-di-GMP in bacterial virulence}

C-di-GMP modulates virulence of animal and plant pathogens ${ }^{12}$. Processes controlled by c-di-GMP include host cell adherence, secretion of virulence factors, cytotoxicity, invasion, resistance to oxidative stress, and modulation of the immune response. Importantly, recent findings have linked c-di-GMP to the most prominent secretion systems for virulence factors including T2SS, T3SS and T6SS ${ }^{52,54,55}$. This opens up the possibility that c-di-GMP interferes with these processes on a more global scale. An emerging example for the importance of c-diGMP in virulence is Clostridium difficile. In contrast to most Gram-positive bacteria C. difficile encodes a large number of enzymes involved in c-di-GMP turnover ${ }^{89}$. In the course of infections, $C$. difficile undergoes a c-di-GMP-mediated switch from a motile to a surface adherent state with cells adhering to the intestinal mucosa via T4P and other adhesins ${ }^{90}$. This transition is mediated by a total of 16 c-di-GMP-responsive riboswitches, 12 of which being OFF switches (Type I) and four being ON switches (type II) ${ }^{91}$. Through these regulatory elements, c-di-GMP controls the expression of flagella, pili, adhesion factors and other virulence 
factors including toxins $\mathrm{Tcd} A$ and $\mathrm{TcdB}$, the main virulence factors of $C$. difficile ${ }^{81,91-94}$ (Figure 4). For example, a collagen binding protein (CBP) and its specific protease are inversely controlled by Type I and II riboswitches, respectively ${ }^{91}$. Expression of the protease at low cdi-GMP concentrations effectively prevents host cell adherence, while expression of the CBP at high c-di-GMP concentrations promotes attachment to host tissue. Thus, c-di-GMPmediated riboswitches control $C$. difficile host colonization by coordinating motility, toxin production, surface adhesion and biofilm formation.

Another example for the prominent role of c-di-GMP in virulence is the 2011 German outbreak of E. coli $\mathrm{O} 104: \mathrm{H} 4$, which caused a unusually high incidence of haemolytic uraemic syndrome (HUS) ${ }^{95}$. The genome of the causative strain showed characteristics of both enterhaemorrhagic E. coli (EHEC) and enteroaggregative E. coli (EAEC) and revealed the presence of a highly expressed diguanylate cyclase $(d g c X)$, which is prevalent in EAEC O104:H4 strains ${ }^{96}$. This indicated that the outbreak strain and EAEC in general produce high levels of c-di-GMP and likely form biofilms in the host. The observation that the $d g c X$ gene is inserted at the attB locus, the integration site for phage lambda, and is flanked by prophage elements, suggested acquisition by horizontal gene transfer. The analysis of E. coli O104:H4 also emphasized the key importance of adaptation and regulatory flexibility of the c-di-GMP network. While strong adherence, together with Shiga-toxin expression, is a key virulence factor of $E$. coli $\mathrm{O} 104: \mathrm{H} 4$, this strain produces curli but is cellulose negative. The authors of this study speculated that the strong pro-inflammatory effect of curli together with the absence of cellulose (which normally counteracts this effect) may facilitate entry into the bloodstream and kidneys where this pathogen can cause life-threatening hemolytic uremic syndrome ${ }^{96}$. Given their unique distribution in bacteria and their importance in bacterial virulence it is not surprising that bacterial CDNs did not go unnoticed by the host's immune system. Recent evidence points to a prominent role for c-di-GMP and c-di-AMP as PAMPs, pathogenassociated molecular patterns that are specifically recognized by the innate immune system of the host (Box 2).

\section{Conclusion and outlook}

Above we have summarized some of the recent findings describing mechanistic and functional aspects of c-di-GMP signaling in bacteria. Although c-di-AMP was discovered more recently, the field is picking up rapidly exposing comparable physiological complexity (Box 2). It is possible that additional CDNs still await their discovery offering even greater signal- 
ing diversity by varying either the nucleotide composition or linkage chemistry. But why are CDNs so prevalent in controlling important biological processes in bacteria? One major advantage of second messenger-based networks over other information transfer systems based on protein-protein interaction might be the ease with which they are able to evolve. For example, recruiting additional cellular processes into an existing c-di-GMP network seems relatively straightforward, considering that c-di-GMP often binds on the surface of pre-existing protein domains with only a few amino acids contributing to ligand affinity and specificity (Figure 5a). Simple recruitment of additional effectors together with the rapid expansion of makers and breakers by gene duplication might thus have predisposed CDN-based regulatory networks for the coordination of global metabolic and behavioral transitions in bacteria.

$\mathrm{CDN}$ based second messengers also offer various advantages in signal transduction. Their rapid cellular diffusion stages an instantaneous and global internal response. At the same time CDNs may act in a highly specific manner either through temporal or spatial control. For example, the combination of DGCs or PDEs with distinct inhibition constants and substrate affinities, respectively, together with effector proteins or RNAs of matching c-di-GMP affinities would permit cells to regulate different processes in a highly specific manner (Figure 5b). Alternatively, spatial organization with DGCs and/or PDEs interacting directly with their respective targets in combination with effective mechanisms isolating individual signaling modules from each other would permit parallel CDN signaling modules with highly specific readouts (Figure 5c). CDNs like c-di-GMP control the expression, activity, stability, localization or interaction of specific proteins $\left(\right.$ e.g. $\left.{ }^{42,61,78,97}\right)$. Moreover, c-di-GMP can control the same biological process at different levels including transcription, translation or allosteric control (Figure 5d) (e.g. ${ }^{52}$ ). Such a multi-layered signaling architecture can impose tight control and continuous evaluation power over strictly unidirectional cellular processes like cell cycle progression or processes with considerable metabolic cost like the motile-sessile switch. It can also provide bacteria with the ability to rapidly sample the environment and to adjust their behavior without the need for de novo protein synthesis. Or it could serve to integrate two distinct processes but at the same time uncouple them if necessary by the use of distinct DGC/PDE modules (Figure 5d). An example of such a process is illustrated by the production of E. coli curli and cellulose (see above). Finally, it could be used to define activity windows for specific cellular processes, for example by sequential expression control (module 1) and inactivation of a downstream effector (module 2), which is either turned off by c-di-GMP or subject to c-di-GMP mediated degradation. 
459 Despite the advances in the CDN field, many important questions remain to be addressed 460 in the future. For example, are there additional CDNs to be included in this emerging signal461 ing paradigm? Which cellular activities do specific CDN networks control and how extensive462 ly do these compounds interfere with basic cellular processes in bacteria? What is the exact 463 architecture of CDN networks and how do they contribute to the highly dynamic behavior of 464 bacterial cells? And how do CDN-based networks integrate with other signaling networks like 465 quorum sensing, phosphorylation cascades or regulation by ppGpp? It is safe to predict that 466 this field of research will continue to provide exciting novel insights into bacterial signaling, 467 growth and behavior.

468

469 


\section{Box 1: Toolkit for CDN analysis}

Several novel approaches were developed in the past years to identify and characterize CDN effector proteins on a global scale. This included affinity pull-downs followed by subsequent mass spectrometry analysis. Trivalent chemical scaffolds with a CDN binding, a biotin sorting, and a crosslinking moiety were used as capture compounds in combination with strepdavidin coated magnetic beads ${ }^{98,99}$. A similar approach used c-di-GMP coated sepharose beads for affinity pull-down ${ }^{100}$. The advantage of these methods is that potential binding proteins can directly be isolated from cell extracts without the need for time consuming fractionation or biochemical purification. Moreover, once specific binding proteins have been identified, such pull-down methods can also be employed for diagnostic purposes in combination with specific antibodies ${ }^{78}$. Both methods were successfully applied with different bacteria including Pseudomonas, Caulobacter, Listeria, Streptomyces or Bdellovibrio ${ }^{42,98,101,102}$. A more indirect approach made use of the complete ORFeome and subsequent testing of cell lysates with a high-throughput binding assay ${ }^{52,103}$. Differential radial capillary action of ligand assay (DRaCALA) was developed to directly mix proteins with (radio)-labeled nucleotide(s) on a nitrocellulose membrane. Upon washing the filters free ligand will diffuse away, while ligand specifically bound by proteins will be immobilized at contact site ${ }^{103}$. Application of these techniques as well as more conventional approaches like Isothermal Titration Calorimetry (ITC) or Microscale Thermophoresis (MST) have led to the identification of a plethora of novel effector proteins $52,55,100,104$.

Several tools and biomarkers were established for in vitro and in vivo analysis of c-diGMP. This includes sensitive high performance liquid chromatography-coupled tandem mass spectrometry (HPLC-MS/MS) to accurately determine the concentration of second messengers in cell extracts ${ }^{105,106}$, fluorescence-based reporters fused to c-di-GMP dependent promoters or riboswitches ${ }^{107-110}$, and a c-di-GMP specific FRET probe that allows direct measurements of c-di-GMP concentrations in individual live cells ${ }^{59,66}$. 


\section{Box 2: CDNs beyond c-di-GMP}

The CDN c-di-AMP was discovered as a ligand bound to the $\mathrm{N}$-terminal domain of the DNA damage-sensing protein DisA of B. subtilis $^{2}$. Biochemical studies identified this domain as diadenylate cylcase (DAC), the founding member of a family of enzymes that converts ATP to c-di-AMP. Specific PDEs associated with DHH-DHHA1 or HD domains hydrolyze cdi-AMP into pApA or AMP ${ }^{111-113}$. C-di-AMP is essential in a variety of different bacteria and any dysregulation causes abnormal phenotypes ${ }^{114,115}$. A recent report suggested that in Listeria monocytogenes this effect is due to overshooting levels of (p)ppGpp, a global second messenger linked to carbon metabolism and nutrient starvation ${ }^{116}$. C-di-AMP is associated with a growing list of cellular functions primarily in gram-positive bacteria. These include cell wall homeostasis ${ }^{115,117-120}$, DNA integrity ${ }^{2,121-123}$, potassium homeostasis ${ }^{104,124-126}$ and osmoprotection $^{127,128}$, gene expression ${ }^{129,130}$, biofilm formation ${ }^{131,132}$, sporulation ${ }^{133}$, metabo$\operatorname{lism}^{102}$, resistance to antibiotics ${ }^{134}$, and, similar to c-di-GMP, cell-mediated adaptive immune response (see below).

c-GMP-AMP is of special interest because it is produced by bacteria and metazoans ${ }^{3,135}$. Bacterial c-GMP-AMP shows 3'-3' linkage and is produced by the dinucleotide synthase DncV originally identified in $V$. cholera ${ }^{3}$. Structural studies revealed that in the first nucleotidyl transfer reaction DncV preferably recognizes ATP and GTP as acceptor and donor nucleotides, respectively ${ }^{136}$. c-GMP-AMP is required for host colonization by $V$. cholera and for exoelectrogenesis in different delta-proteobacteria ${ }^{137,138}$. Mammalian c-GMP-AMP (2'-3') (cGAMP) has adopted a prominent role in a vertebrate innate immunity pathway responsible for surveillance of cytoplasmic DNA ${ }^{139}$. cGAMP is synthesized by the cGAMP synthase (cGAS), which is activated in response to binding cytoplasmic DNA ${ }^{140,141}$. cGAMP binds to and activates the host receptor STING which in turn recruits TANK-binding kinase 1 (TBK1) to phosphorylate IFN regulatory factor 3 (IRF3), ultimately leading to type I interferon (IFN) production. Evolutionary studies recently revealed that the cGAS-STING function is conserved in anemone, which diverged from the human lineage more than 500 million years ago. Because Anemone cGAS produces a bacteria-like 3'-3' linked CDN that is recognized by Anemone STING, it was proposed that cGAMP (2'-3') is a recent vertebrate innovation and that during evolution the protein components of this pathway remained structurally conserved, while chemical changes in the second messenger were driving functional innovation ${ }^{142}$.

Recent evidence suggests that c-di-GMP and c-di-AMP, secreted or released outside bacteria, are also sensed by STING (stimulator of interferon genes) thereby converging with the cGAS-cGAMP cytosolic DNA surveillance pathway ${ }^{143-145}$. Interestingly, bacteria seem to 
532 have evolved strategies to dampen IFN production by avoiding STING activation. Group B

533 Streptococcus was recently shown to express an ectonucleotidase, CdnP, which hydrolyzes

534 extracellular bacterial c-di-AMP to attenuate the cGAS-STING axis ${ }^{146}$.

535

536

537

538 
540 Figure 1: Components of the c-di-GMP signaling network. (a) Principles of c-di-GMP signaling. Enzymatic reactions are depicted as grey arrows. GGDEF, EAL and HD-GYP represent conserved catalytic domains of diguanylate cyclases and phosphodiesterases, respectively. (b) Schematic of DGC activation. Upper panel: phosphorylation-dependent activation of PleD from C. crescentus. Receiver-domains (Rec) are shown in green and GGDEFdomains in orange. Phosphorylation-induced dimerization of Rec-domain stem leads to dimerization and activation of GGDEF-domains. Lower panel: metal-dependent activation of $E$. coli DgcZ. DgcZ is a constitutive dimer. Zinc-depletion from the CZB-domain leads to competent positioning of the GGDEF-domains. (c) Structure of the zinc-binding diguanylate cyclase DgcZ from E. coli (PDB: 4H54) ${ }^{22}$. GGDEF-domains (orange) and zinc-binding CZBdomain (grey) are highlighted. Zinc metal ions are depicted as red spheres. C-di-GMP (magenta) binding to antipodal inhibitory I-sites (IP \& IP') and GTP $\alpha$ S (green) binding to active sites (A \& A') are shown. (d) Overlay of the EAL domains of the phosphodiesterase PdeL in the tight, substrate-bound (blue; PDB: 4LJ3) and relaxed (apo) conformation (grey, PDB: $4 \mathrm{LYK})^{27}$. Inlet: zoom-in of the active site and conserved loop 6 region. The loop 6 conformations in the relaxed, apo (yellow) and tight, c-di-GMP-bound (orange) dimer are indicated. Yellow and magenta spheres indicate the positions of $\mathrm{Mg}^{2+}$-ions in the relaxed and tight protein conformations, respectively. The conserved double-aspartic acid motif (D262, D263) and anchoring glutamate (E235), which determine the structural arrangement of loop 6 are highlighted. (e) Structure of Rhodobacter sphaeroides cellulose synthase complex with the BcsA subunit (green), its C-terminal PilZ-domain (magenta) and the BscB subunit (grey) (PDB: 4P02) ${ }^{46}$. The cytoplasmic membrane is outlined in grey. A dimer of c-di-GMP bound to the PilZ domain is marked.

Figure 2: Role of c-di-GMP in $C$. crescentus pole morphogenesis and cell cycle progression. A schematic of the Caulobacter cell cycle is shown with flagellated swarmer cells (SW, G1-phase), stalked cells (ST, S-phase) and predivisional cells (division) indicated. Polar organelles (flagellum, pili, stalk and holdfast) of individual cell types are marked. The replication status of the circular chromosome is indicated schematically with SW cells being replication silent while chromosome replication initiates in ST cells. Cell type-specific levels of c-diGMP are as indicated. The subcellular localization of the DGC PleD, the PDE PdeA, the flagellar placement protein TipF and the sensor histidine kinases PleC, DivJ and CckA are 
marked at individual stages of the cell cycle. Individual panels highlight stage-specific processes at the stalked and flagellated poles. Autophosphorylation of histidine kinases (DivJ, CckA) and phosphotransfer to response regulators (PleD, CtrA) are indicated. a) Flagellar assembly. TipF binds to c-di-GMP to localize to the flagellated pole, where it recruits flagellar components PflI and FliG to initiate flagellar assembly. b) Low c-di-GMP levels at the flagellated pole of dividing cells and in SW cells promote TipF degradation by the ClpXP protease and CckA kinase activity. The CckA kinase activates the CtrA replication initiation inhibitor by phosphorylation via the P-transfer protein ChpT. PdeA and as yet unidentified PDE(s) contribute to the reduction of the c-di-GMP concentration at this cell cycle stage. c) Degradation of PdeA and CtrA by the ClpXP protease during the SW-to-ST transition and at the stalked pole of the dividing cell. PleD and as yet unidentified DGC(s) contribute to the upshift of cdi-GMP upon entry into S-phase and in the predivisional cell. Activation of the protease adaptor PopA by c-di-GMP leads to the degradation of CtrA. d) Inactivation of CtrA by the CckA phosphatase during the SW-to-ST transition and at the stalked pole of the dividing cell. PleD and as yet unidentified DGC(s) contribute to the upshift of c-di-GMP upon entry into S-phase and in the predivisional cell. Binding of c-di-GMP causes the switch of the CckA histidine kinase from its default kinase to the S-phase-specific phosphatase state.

Figure 3: Role of c-di-GMP in biofilm formation and dispersal. Bacterial surface attachment, biofilm formation and dispersal are indicated schematically in the central panel. (a) cdi-GMP-mediated control of flagellar motility in E. coli. DGCs (orange), PDE (blue) and the c-di-GMP effector YcgR (purple) are highlighted. YcgR interacts with and curbs the flagellar motor upon binding of c-di-GMP. PdeH adopts a key role to inactivate YcgR by keeping c-diGMP levels low thereby enabling motor function. (b) c-di-GMP-dependent production of amyloid curli fibers and cellulose in E. coli. The global (DgcE/PdeH) and local (DgcM/PdeR) modules of DGCs and PDEs controlling $\operatorname{csg} D$ transcription are indicated. DgcM/PdeR sense the global concentration of c-di-GMP and, in response, activate the transcription factor MlrA. The global transcription factor $\mathrm{CsgD}$ then activates the expression of curli components and of $\mathrm{DgcC}$, the main activator of the cellulose synthase complex. (c) c-di-GMP-mediated synthesis of poly-beta-1,6-N-acetyl-glucosamine (GlcNAc) in E. coli. The Csr global regulatory system co-regulates the pga genes encoding components of the GlcNAc synthesis machinery (PgaAD) and $d g c T$ and $d g c Z$ encoding two DGCs (orange) responsible for the allosteric activation of PgaCD. The histidine kinase BarA is stimulated by short-chain fatty acids and through the phosphorylation of the response regulator UvrY, activates the expression of two small RNAs, 
CsrB and $\mathrm{CsrC}$, which in turn antagonize the translation inhibitor CsrA. (d) Biofilm escape mechanism in P. fluorescens Pf0-1. The LapA surface protein mediates $P$. fluorescens surface adhesion and contributes to the stabilization of biofilms. Under phosphate starvation conditions LapA is degraded by the periplasmic protease LapG resulting in biofilm escape. If enough phosphate is available, LapG is sequestered by its partner LapD in its c-di-GMP bound conformation. When phosphate becomes limited the RapA PDE is expressed through the phosphate control system Pst/PhoRB, leading to a drop of c-di-GMP, a conformational change of apo-LapD and the release of the protease.

Figure 4: Role of c-di-GMP in virulence of Clostridium difficile. C. difficile virulence is regulated by c-di-GMP-specific riboswitches. Type-I riboswitches and type-II riboswitches control the expression of factors involved in motility, surface attachment and virulence. TypeI riboswitches (OFF-switches) inhibit translation upon c-di-GMP binding, while type-IIriboswitches (ON-switches) promote translation of target genes when bound to c-di-GMP. Increasing levels of c-di-GMP stimulate the expression of adhesion factors such as type-4-pili (T4P) and collagen-binding proteins (CBP) and inhibit the expression of flagellar genes and the CBP protease. The gene encoding the sigma factor SigD is co-regulated with flagellar genes. Thus, when the c-di-GMP concentration is low, cells not only express motility and anti-adhesion genes, but also express the SigD-dependent Cdiff toxins TcdA and TcdB.

Figure 5: General concepts of c-di-GMP signaling modules. Effectors (E) (c-di-GMP binding proteins), diguanylate cyclases (DGC) and phosphodiesterases (PDE) are labeled. c-diGMP molecules are indicated as black circles or as spatial gradient in (c). (a) Evolutionary diagram of recruiting cellular processes into an existing CDN network. Minor modifications of the surface of a specific protein can mediate specific binding of c-di-GMP, which in turn can modulate the protein's activity, stability or interaction with a partner. (b) and (c) Network architecture involved in pathway-specific signaling. C-di-GMP-dependent processes can be specifically regulated by temporal (b) or spatial (c) separation. Temporal regulation relies on effector proteins with different ligand affinities and on DGCs and/or PDEs with specific inhibition and activation constants, respectively. This allows establishing precise cellular thresholds of c-di-GMP thereby activating specific downstream effectors and pathways. Spatially separated signaling relies on some form of compartmentalization, for example with a specific 
DGC/PDEs module interacting with its specific effector. To avoid unwanted crosstalk with other effectors and cellular pathways, spatially confined modules need to be effectively insulated. This can occur via the action of the module-specific PDE or by a general cellular PDE that restrains leakage of c-di-GMP. (d) C-di-GMP can interfere with the same biological process at different levels of control. E.g. c-di-GMP can control gene expression (transcription/translation) or control the activity of one of the resulting proteins as indicated. Expression and allosteric control can be mediated by the same module comprising a DGC and PDE (arrows) or can be modulate independently by different DGC/PDEs modules.

\section{Acknowledgements:}

This work was supported by grants of the Swiss National Science Foundation grant (310030B_147090) to U.J. and by an ERC Advanced Research Grant (322809) to U.J.

\section{References}

1. Ross, P. et al. Regulation of cellulose synthesis in Acetobacter xylinum by cyclic diguanylic acid. Nature 325, 279-281 (1987).

2. Witte, G., Hartung, S., Büttner, K. \& Hopfner, K.-P. Structural Biochemistry of a Bacterial Checkpoint Protein Reveals Diadenylate Cyclase Activity Regulated by DNA Recombination Intermediates. Molecular Cell 30, 167-178 (2008).

3. Davies, B. W., Bogard, R. W., Young, T. S. \& Mekalanos, J. J. Coordinated regulation of accessory genetic elements produces cyclic di-nucleotides for V. cholerae virulence. Cell 149, 358-370 (2012).

4. Hornung, V., Hartmann, R., Ablasser, A. \& Hopfner, K.-P. OAS proteins and cGAS: unifying concepts in sensing and responding to cytosolic nucleic acids. Nature Reviews Immunology 14, 521-528 (2014).

5. Chan, C. et al. Structural basis of activity and allosteric control of diguanylate cyclase. Proceedings of the National Academy of Sciences of the United States of America 101, 17084-17089 (2004).

6. Kranzusch, P. J. et al. Structure-guided reprogramming of human cGAS dinucleotide linkage specificity. Cell 158, 1011-1021 (2014).

7. Lori, C. et al. Cyclic di-GMP acts as a cell cycle oscillator to drive chromosome replication. Nature 523, 236-239 (2015).

8. Srivastava, D. \& Waters, C. M. A tangled web: regulatory connections between quorum sensing and cyclic Di-GMP. Journal of Bacteriology 194, 4485-4493 (2012).

9. Gupta, K. R., Kasetty, S. \& Chatterji, D. Novel functions of (p)ppGpp and Cyclic diGMP in mycobacterial physiology revealed by phenotype microarray analysis of wild-type and isogenic strains of Mycobacterium smegmatis. Applied and Environmental Microbiology 81, 2571-2578 (2015).

10. An, S.-Q. et al. A cyclic GMP-dependent signalling pathway regulates bacterial phytopathogenesis. The EMBO Journal 32, 2430-2438 (2013). 
11. Almblad, H. et al. The Cyclic AMP-Vfr Signaling Pathway in Pseudomonas aeruginosa Is Inhibited by Cyclic Di-GMP. Journal of Bacteriology 197, 2190-2200 (2015).

12. Romling, U., Galperin, M. Y., Gomelsky, M. Cyclic di-GMP: the first 25 years of a universal bacterial second messenger. Microbiology and Molecular Biology Reviews 77, 1-52 (2013).

13. Corrigan, R. M. \& Gründling, A. Cyclic di-AMP: another second messenger enters the fray. Nature reviews Microbiology 11, 513-524 (2013).

14. Cai, X., Chiu, Y.-H. \& Chen, Z. J. The cGAS-cGAMP-STING pathway of cytosolic DNA sensing and signaling. Molecular Cell 54, 289-296 (2014).

15. Danilchanka, O. \& Mekalanos, J. J. Cyclic Dinucleotides andthe Innate Immune Response. Cell 154, 962-970 (2013).

16. Kalia, D. et al. Nucleotide, c-di-GMP, c-di-AMP, cGMP, cAMP, (p)ppGpp signaling in bacteria and implications in pathogenesis. Chem Soc Rev 42, 305-341 (2013).

17. Schirmer, T. C-di-GMP Synthesis: Structural Aspects of Evolution, Catalysis and Regulation. Journal of Molecular Biology (2016). doi:10.1016/j.jmb.2016.07.023

18. Gentner, M., Allan, M. G., Zaehringer, F., Schirmer, T. \& Grzesiek, S. Oligomer Formation of the Bacterial Second Messenger c-di-GMP: Reaction Rates and Equilibrium Constants Indicate a Monomeric State at Physiological Concentrations. Journal of the American Chemical Society 134, 1019-1029 (2012).

19. Wassmann, P. et al. Structure of BeF3--Modified Response Regulator PleD: Implications for Diguanylate Cyclase Activation, Catalysis, and Feedback Inhibition. Structure 15, 915-927 (2007).

20. Paul, R. et al. Activation of the diguanylate cyclase PleD by phosphorylationmediated dimerization. The Journal of biological chemistry 282, 29170-29177 (2007).

21. Christen, B. et al. Allosteric Control of Cyclic di-GMP Signaling. Journal of Biological Chemistry 281, 32015-32024 (2006).

22. Zähringer, F., Lacanna, E., Jenal, U., Schirmer, T. \& Boehm, A. Structure and signaling mechanism of a zinc-sensory diguanylate cyclase. Structure 21, 1149-1157 (2013).

23. De, N. et al. Phosphorylation-Independent Regulation of the Diguanylate Cyclase WspR. PLoS Biology 6, e67 (2008).

24. Dahlstrom, K. M., Giglio, K. M., Sondermann, H. \& O’Toole, G. A. The Inhibitory Site of a Diguanylate Cyclase Is a Necessary Element for Interaction and Signaling with an Effector Protein. Journal of Bacteriology 198, 1595-1603 (2016).

25. Christen, M., Christen, B., Folcher, M., Schauerte, A. \& Jenal, U. Identification and Characterization of a Cyclic di-GMP-specific Phosphodiesterase and Its Allosteric Control by GTP. Journal of Biological Chemistry 280, 30829-30837 (2005).

26. Barends, T. R. M. et al. Structure and mechanism of a bacterial light-regulated cyclic nucleotide phosphodiesterase. Nature 459, 1015-1018 (2009).

27. Sundriyal, A. et al. Inherent regulation of EAL domain-catalyzed hydrolysis of second messenger cyclic di-GMP. Journal of Biological Chemistry 289, 6978-6990 (2014).

28. Winkler, A. et al. Characterization of elements involved in allosteric light regulation of phosphodiesterase activity by comparison of different functional BlrP1 states. Journal of Molecular Biology 426, 853-868 (2014).

29. Rao, F. et al. The Functional Role of a Conserved Loop in EAL Domain-Based Cyclic di-GMP-Specific Phosphodiesterase. Journal of Bacteriology 191, 4722-4731 (2009).

30. Navarro, M. V. A. S. et al. Structural Basis for c-di-GMP-Mediated Inside-Out Sig- 
naling Controlling Periplasmic Proteolysis. PLoS Biology 9, e1000588 (2011).

31. Ryan, R. P., Fouhy, Y. \& Lucey, J. F. Cell-cell signaling in Xanthomonas campestris involves an HD-GYP domain protein that functions in cyclic di-GMP turnover. Proceedings of the National Academy of Sciences of the United States of America 103, 6712-17 (2006).

32. Bellini, D. et al. Crystal structure of an HD-GYP domain cyclic-di-GMP phosphodiesterase reveals an enzyme with a novel trinuclear catalytic iron centre. Molecular Microbiology 91, 26-38 (2013).

33. Orr, M. W. et al. Oligoribonuclease is the primary degradative enzyme for $\mathrm{pGpG}$ in Pseudomonas aeruginosa that is required for cyclic-di-GMP turnover. Proceedings of the National Academy of Sciences 112, E5048-57 (2015).

34. Cohen, D. et al. Oligoribonuclease is a central feature of cyclic diguanylate signaling in Pseudomonas aeruginosa. Proceedings of the National Academy of Sciences 112, 11359-11364 (2015).

35. Hengge, R. et al. Systematic Nomenclature for GGDEF and EAL DomainContaining Cyclic Di-GMP Turnover Proteins of Escherichia coli. Journal of Bacteriology 198, 7-11 (2015).

36. Boehm, A. et al. Second Messenger-Mediated Adjustment of Bacterial Swimming Velocity. Cell 141, 107-116 (2010).

37. Reinders, A. et al. Expression and Genetic Activation of Cyclic Di-GMP-Specific Phosphodiesterases in Escherichia coli. Journal of Bacteriology 198, 448-462 (2016).

38. Tuckerman, J. R. et al. An Oxygen-Sensing Diguanylate Cyclase and Phosphodiesterase Couple for c-di-GMP Control. Biochemistry 48, 9764-9774 (2009).

39. Lindenberg, S., Klauck, G., Pesavento, C., Klauck, E. \& Hengge, R. The EAL domain protein YciR acts as a trigger enzyme in a c-di-GMP signalling cascadein $\mathrm{E}$. coli biofilm control. The EMBO Journal 32, 2001-2014 (2013).

40. Chou, S.-H. \& Galperin, M. Y. Diversity of Cyclic Di-GMP-Binding Proteins and Mechanisms. Journal of Bacteriology 198, 32-46 (2016).

41. Hengge, R. Cyclic-di-GMP Reaches Out into the Bacterial RNA World. Science Signaling 3, pe44-pe44 (2010).

42. Tschowri, N. et al. Tetrameric c-di-GMP Mediates Effective Transcription Factor Dimerizationto Control Streptomyces Development. Cell 158, 1136-1147 (2014).

43. Habazettl, J., Allan, M. G., Jenal, U. \& Grzesiek, S. Solution structure of the PilZ domain protein PA4608 complex with cyclic di-GMP identifies charge clustering as molecular readout. Journal of Biological Chemistry 286, 14304-14314 (2011).

44. Schumacher, M. A. \& Zeng, W. Structures of the activator of K. pneumonia biofilm formation, MrkH, indicates PilZ domains involved in c-di-GMP and DNA binding. Proceedings of the National Academy of Sciences (2016). doi:10.1073/pnas. 1607503113

45. Duerig, A. et al. Second messenger-mediated spatiotemporal control of protein degradation regulates bacterial cell cycle progression. Genes \& Development 23, 93-104 (2009).

46. Morgan, J. L. W., McNamara, J. T. \& Zimmer, J. Mechanism of activation of bacterial cellulose synthase by cyclic di-GMP. Nat Struct Mol Biol 21, 489-496 (2014).

47. An, S.-Q. et al. Novel cyclic di-GMP effectors of the YajQ protein family control bacterial virulence. PLoS Pathogens 10, e1004429 (2014).

48. Fazli, M. et al. The CRP/FNR family protein Bcam1349 is a c-di-GMP effector that regulates biofilm formation in the respiratory pathogen Burkholderia cenocepacia. Molecular Microbiology 82, 327-341 (2011).

49. Krasteva, P. V. et al. Vibrio cholerae VpsT Regulates Matrix Production and Motility by Directly Sensing Cyclic di-GMP. Science 327, 866-868 (2010). 
50. Matsuyama, B. Y. et al. Mechanistic insights into c-di-GMP-dependent control of the biofilm regulator FleQ from Pseudomonas aeruginosa. Proceedings of the National Academy of Sciences 113, E209-18 (2016).

51. Baraquet, C. \& Harwood, C. S. Cyclic diguanosine monophosphate represses bacterial flagella synthesis by interacting with the Walker A motif of the enhancer-binding protein FleQ. Proceedings of the National Academy of Sciences 110, 18478-18483 (2013).

52. Roelofs, K. G. et al. Systematic Identification of Cyclic-di-GMP Binding Proteins in Vibrio cholerae Reveals a Novel Class of Cyclic-di-GMP-Binding ATPases Associated with Type II Secretion Systems. PLoS Pathogens 11, e1005232 (2015).

53. Jones, C. J. et al. C-di-GMP Regulates Motile to Sessile Transition by Modulating MshA Pili Biogenesis and Near-Surface Motility Behavior in Vibrio cholerae. PLoS Pathogens 11, e1005068 (2015).

54. Moscoso, J. A., Mikkelsen, H., Heeb, S., Williams, P. \& Filloux, A. The Pseudomonas aeruginosa sensor RetS switches Type III and Type VI secretion via c-di-GMP signalling. Environmental Microbiology 13, 3128-3138 (2011).

55. Trampari, E. et al. Bacterial rotary export ATPases are allosterically regulated by the nucleotide second messenger cyclic-di-GMP. Journal of Biological Chemistry 290, 24470-24483 (2015).

56. Kirkpatrick, C. L. \& Viollier, P. H. Decoding Caulobacter development. FEMS Microbiology Reviews 36, 193-205 (2012).

57. Abel, S. et al. Regulatory Cohesion of Cell Cycle and Cell Differentiation through InterlinkedPhosphorylation and Second Messenger Networks. Molecular Cell 43, 550560 (2011).

58. Abel, S. et al. Bi-modal Distribution of the Second Messenger c-di-GMP Controls Cell Fate and Asymmetry during the Caulobacter Cell Cycle. PLoS genetics 9, e1003744 (2013).

59. Christen, M. et al. Asymmetrical Distribution of the Second Messenger c-di-GMP upon Bacterial Cell Division. Science 328, 1295-1297 (2010).

60. Paul, R. et al. Allosteric Regulation of Histidine Kinases by Their Cognate Response Regulator Determines Cell Fate. Cell 133, 452-461 (2008).

61. Davis, N. J. et al. De- and repolarization mechanism of flagellar morphogenesis during a bacterial cell cycle. Genes \& Development 27, 2049-2062 (2013).

62. Ozaki, S. et al. Activation and polar sequestration of PopA, a c-di-GMP effector protein involved in Caulobacter crescentuscell cycle control. Molecular Microbiology 94, 580-594 (2014).

63. Smith, S. C. et al. Cell cycle-dependent adaptor complex for ClpXP-mediated proteolysis directly integrates phosphorylation and second messenger signals. Proceedings of the National Academy of Sciences 111, 14229-14234 (2014).

64. Chen, Y. E. et al. Spatial gradient of protein phosphorylation underlies replicative asymmetry in a bacterium. Proceedings of the National Academy of Sciences 108, 1052-1057 (2011).

65. Tsokos, C. G., Perchuk, B. S. \& Laub, M. T. A Dynamic Complex of Signaling Proteins Uses Polar Localization to Regulate Cell-Fate Asymmetry in Caulobacter crescentus. Developmental Cell 20, 329-341 (2011).

66. Kulasekara, B. R. et al. c-di-GMP heterogeneity is generated by the chemotaxis machinery to regulate flagellar motility. eLife 2, e01402 (2013).

67. Bush, M. J., Tschowri, N., Schlimpert, S., Flärdh, K. \& Buttner, M. J. c-di-GMP signalling and the regulation of developmental transitions in streptomycetes. Nature reviews Microbiology 13, 749-760 (2015).

68. Petters, T. et al. The orphan histidine protein kinase SgmT is a c-di-GMP receptor 
and regulates composition of the extracellular matrix together with the orphan DNA binding response regulator DigR in Myxococcus xanthus. Molecular Microbiology 84, 147-165 (2012).

69. Hobley, L. et al. Discrete cyclic di-GMP-dependent control of bacterial predation versus axenic growth in Bdellovibrio bacteriovorus. PLoS Pathogens 8, e1002493 (2012).

70. Enomoto, G., Ni-Ni-Win, Narikawa, R. \& Ikeuchi, M. Three cyanobacteriochromes work together to form a light color-sensitive input system for c-di-GMP signaling of cell aggregation. Proceedings of the National Academy of Sciences 112, 8082-8087 (2015).

71. Valentini, M. \& Filloux, A. Biofilms and Cyclic di-GMP (c-di-GMP) Signaling: Lessons from Pseudomonas aeruginosa and Other Bacteria. Journal of Biological Chemistry 291, 12547-12555 (2016).

72. Russell, M. H. et al. Integration of the Second Messenger c-di-GMP into the Chemotactic Signaling Pathway. mBio 4, e00001-13-e00001-13 (2013).

73. Paul, K., Nieto, V., Carlquist, W. C., Blair, D. F. \& Harshey, R. M. The c-di-GMP Binding Protein YcgR Controls Flagellar Motor Direction and Speed to Affect Chemotaxis by a "Backstop Brake" Mechanism. Molecular Cell 38, 128-139 (2010).

74. Chen, Y., Chai, Y., Guo, J.-H. \& Losick, R. Evidence for cyclic Di-GMP-mediated signaling in Bacillus subtilis. Journal of Bacteriology 194, 5080-5090 (2012).

75. Pultz, I. S. et al. The response threshold of SalmonellaPilZ domain proteins is determined by their binding affinities for c-di-GMP. Molecular Microbiology 86, 14241440 (2012).

76. Park, J. H. et al. The cabABC Operon Essential for Biofilm and Rugose Colony Development in Vibrio vulnificus. PLoS Pathogens 11, e1005192 (2015).

77. Kariisa, A. T., Weeks, K. \& Tamayo, R. The RNA Domain Vc1 Regulates Downstream Gene Expression in Response to Cyclic Diguanylate in Vibrio cholerae. PLoS ONE 11, e0148478 (2016).

78. Steiner, S., Lori, C., Boehm, A. \& Jenal, U. Allosteric activation of exopolysaccharide synthesis through cyclic di-GMP-stimulated protein-protein interaction. The EMBO Journal 32, 354-368 (2013).

79. Skotnicka, D. et al. Cyclic Di-GMP Regulates Type IV Pilus-Dependent Motility in Myxococcus xanthus. Journal of Bacteriology 198, 77-90 (2016).

80. Kazmierczak, B. I., Lebron, M. B. \& Murray, T. S. Analysis of FimX, a phosphodiesterase that governs twitching motility in Pseudomonas aeruginosa. Molecular Microbiology 60, 1026-1043 (2006).

81. Bordeleau, E. et al. Cyclic di-GMP riboswitch-regulated type IV pili contribute to aggregation of Clostridium difficile. Journal of Bacteriology 197, 819-832 (2015).

82. Serra, D. O., Richter, A. M., Klauck, G., Mika, F. \& Hengge, R. Microanatomy at Cellular Resolution and Spatial Order of Physiological Differentiation in a Bacterial Biofilm. mBio 4, e00103-13-e00103-13 (2013).

83. Pesavento, C. et al. Inverse regulatory coordination of motility and curli-mediated adhesion in Escherichia coli. Genes \& Development 22, 2434-2446 (2008).

84. Vakulskas, C. A., Potts, A. H., Babitzke, P., Ahmer, B. M. M. \& Romeo, T. Regulation of bacterial virulence by Csr (Rsm) systems. Microbiology and molecular biology reviews 79, 193-224 (2015).

85. Chua, S. L. et al. In vitro and in vivo generation and characterization of Pseudomonas aeruginosa biofilm-dispersed cells via c-di-GMP manipulation. Nature Protocols 10, 1165-1180 (2015).

86. Chatterjee, D. et al. Mechanistic insight into the conserved allosteric regulation of 
periplasmic proteolysis by the signaling molecule cyclic-di-GMP. eLife 3, e 03650 (2014).

87. Malone, J. G. et al. YfiBNR Mediates Cyclic di-GMP Dependent Small Colony Variant Formation and Persistence in Pseudomonas aeruginosa. PLoS Pathogens 6, e1000804 (2010).

88. Blanka, A. et al. Constitutive production of c-di-GMP is associated with mutations in a variant of Pseudomonas aeruginosa with altered membrane composition. Science Signaling 8, ra36 (2015).

89. Bordeleau, E., Fortier, L.-C., Malouin, F. \& Burrus, V. c-di-GMP turn-over in Clostridium difficile is controlled by a plethora of diguanylate cyclases and phosphodiesterases. PLoS genetics 7, e1002039 (2011).

90. Purcell, E. B., McKee, R. W., McBride, S. M., Waters, C. M. \& Tamayo, R. Cyclic diguanylate inversely regulates motility and aggregation in Clostridium difficile. Journal of Bacteriology 194, 3307-3316 (2012).

91. Soutourina, O. A. et al. Genome-wide identification of regulatory RNAs in the human pathogen Clostridium difficile. PLoS genetics 9, e1003493 (2013).

92. Purcell, E. B., McKee, R. W., Bordeleau, E., Burrus, V. \& Tamayo, R. Regulation of Type IV Pili Contributes to Surface Behaviors of Historical and Epidemic Strains of Clostridium difficile. Journal of Bacteriology 198, 565-577 (2015).

93. McKee, R. W., Mangalea, M. R., Purcell, E. B., Borchardt, E. K. \& Tamayo, R. The second messenger cyclic Di-GMP regulates Clostridium difficile toxin production by controlling expression of sigD. Journal of Bacteriology 195, 5174-5185 (2013).

94. Peltier, J. et al. Cyclic diGMP regulates production of sortase substrates of Clostridium difficile and their surface exposure through $\mathrm{ZmpI}$ protease-mediated cleavage. Journal of Biological Chemistry 290, 24453-24469 (2015).

95. Buchholz, U. et al. German outbreak of Escherichia coli O104:H4 associated with sprouts. N. Engl. J. Med. 365, 1763-1770 (2011).

96. Richter, A. M., Povolotsky, T. L., Wieler, L. H. \& Hengge, R. Cyclic-di-GMP signalling and biofilm-related properties of the Shiga toxin-producing 2011 German outbreak Escherichia coli O104:H4. EMBO Molecular Medicine 6, 1622-1637 (2014).

97. Lee, E. R., Baker, J. L., Weinberg, Z., Sudarsan, N. \& Breaker, R. R. An allosteric self-splicing ribozyme triggered by a bacterial second messenger. Science 329, 845848 (2010).

98. Nesper, J., Reinders, A., Glatter, T., Schmidt, A. \& Jenal, U. A novel capture compound for the identification and analysis of cyclic di-GMP binding proteins. Journal of proteomics $\mathbf{7 5}$, 4874-4878 (2012).

99. Laventie, B.-J. et al. Capture compound mass spectrometry--a powerful tool to identify novel c-di-GMP effector proteins. $J$ Vis $\operatorname{Exp}(2015)$. doi:10.3791/51404

100. Düvel, J. et al. Journal of Microbiological Methods. Journal of Microbiological Methods 88, 229-236 (2012).

101. Rotem, O. et al. An Extended Cyclic Di-GMP Network in the Predatory Bacterium Bdellovibrio bacteriovorus. Journal of Bacteriology 198, 127-137 (2016).

102. Sureka, K. et al. The cyclic dinucleotide c-di-AMP is an allosteric regulator of metabolic enzyme function. Cell 158, 1389-1401 (2014).

103. Roelofs, K. G., Wang, J. \& Sintim, H. O. Differential radial capillary action of ligand assay for high-throughput detection of protein-metabolite interactions. Proceedings of the National Academy of Sciences 108, 15528-33 (2011).

104. Corrigan, R. M. et al. Systematic identification of conserved bacterial c-di-AMP receptor proteins. Proceedings of the National Academy of Sciences 110, 9084-9089 (2013).

105. Spangler, C., Böhm, A., Jenal, U., Seifert, R. \& Kaever, V. Journal of Microbiologi- 
cal Methods. Journal of Microbiological Methods 81, 226-231 (2010).

106. Burhenne, H. \& Kaever, V. Quantification of cyclic dinucleotides by reversed-phase LC-MS/MS. Methods in molecular biology (Clifton, N.J.) 1016, 27-37 (2013).

107. Pawar, S. V. et al. Novel genetic tools to tackle c-di-GMP-dependent signalling in Pseudomonas aeruginosa. J. Appl. Microbiol. 120, 205-217 (2016).

108. Zhou, H. et al. Characterization of a natural triple-tandem c-di-GMP riboswitch and application of the riboswitch-based dual-fluorescence reporter. Sci Rep 6, 20871 (2016).

109. Kellenberger, C. A., Wilson, S. C., Sales-Lee, J. \& Hammond, M. C. RNA-based fluorescent biosensors for live cell imaging of second messengers cyclic di-GMP and cyclic AMP-GMP. Journal of the American Chemical Society 135, 4906-4909 (2013).

110. Rybtke, M. T. et al. Fluorescence-Based Reporter for Gauging Cyclic Di-GMP Levels in Pseudomonas aeruginosa. Applied and Environmental Microbiology 78, 50605069 (2012).

111. He, Q. et al. Structural and Biochemical Insight into the Mechanism of Rv2837c from Mycobacterium tuberculosis as a c-di-NMP Phosphodiesterase. Journal of Biological Chemistry 291, 3668-3681 (2016).

112. Huynh, T. N. et al. An HD-domain phosphodiesterase mediates cooperative hydrolysis of c-di-AMP to affect bacterial growth and virulence. Proceedings of the National Academy of Sciences 112, E747-E756 (2015).

113. Bai, Y. et al. Two DHH subfamily 1 proteins in Streptococcus pneumoniae possess cyclic di-AMP phosphodiesterase activity and affect bacterial growth and virulence. Journal of Bacteriology 195, 5123-5132 (2013).

114. Mehne, F. M. P. et al. Cyclic di-AMP homeostasis in bacillus subtilis: both lack and high level accumulation of the nucleotide are detrimental for cell growth. Journal of Biological Chemistry 288, 2004-2017 (2013).

115. Witte, C. E. et al. Cyclic di-AMP is critical for Listeria monocytogenes growth, cell wall homeostasis, and establishment of infection. mBio 4, e00282-13 (2013).

116. Whiteley, A. T., Pollock, A. J. \& Portnoy, D. A. The PAMP c-di-AMP Is Essential for Listeria monocytogenes Growth in Rich but Not Minimal Media due to a Toxic Increase in (p)ppGpp. Cell Host \& Microbe 17, 788-798 (2015).

117. Kaplan Zeevi, M. et al. Listeria monocytogenes multidrug resistance transporters and cyclic di-AMP, which contribute to type I interferon induction, play a role in cell wall stress. Journal of Bacteriology 195, 5250-5261 (2013).

118. Zhu, Y. et al. Cyclic-di-AMP synthesis by the diadenylate cyclase CdaA is modulated by the peptidoglycan biosynthesis enzyme GlmM in Lactococcus lactis. Molecular Microbiology 99, 1015-1027 (2016).

119. Luo, Y. \& Helmann, J. D. Analysis of the role of Bacillus subtilis $\sigma \mathrm{M}$ in $\beta$-lactam resistance reveals an essential role for c-di-AMP in peptidoglycan homeostasis. Molecular Microbiology 83, 623-639 (2012).

120. Corrigan, R. M., Abbott, J. C., Burhenne, H., Kaever, V. \& Gründling, A. c-di-AMP Is a New Second Messenger in Staphylococcus aureus with a Role in Controlling Cell Size and Envelope Stress. PLoS Pathogens 7, e1002217 (2011).

121. Oppenheimer-Shaanan, Y., Wexselblatt, E., Katzhendler, J., Yavin, E. \& BenYehuda, S. c-di-AMP reports DNA integrity during sporulation in Bacillus subtilis. EMBO reports 12, 594-601 (2011).

122. Gándara, C. \& Alonso, J. C. DisA and c-di-AMP act at the intersection between DNA-damage response and stress homeostasis in exponentially growing Bacillus subtilis cells. DNA Repair 27, 1-8 (2015).

123. Zhang, L. \& He, Z.-G. Radiation-sensitive gene A (RadA) targets DisA, DNA integ- 
rity scanning protein A, to negatively affect cyclic Di-AMP synthesis activity in Mycobacterium smegmatis. Journal of Biological Chemistry 288, 22426-22436 (2013).

124. Chin, K.-H. et al. Structural Insights into the Distinct Binding Mode of Cyclic DiAMP with SaCpaA_RCK. Biochemistry 54, 4936-4951 (2015).

125. Kim, H. et al. Structural Studies of Potassium Transport Protein KtrA Regulator of Conductance of K+ (RCK) C Domain in Complex with Cyclic Diadenosine Monophosphate (c-di-AMP). Journal of Biological Chemistry 290, 16393-16402 (2015).

126. Moscoso, J. A. et al. Binding of Cyclic Di-AMP to the Staphylococcus aureus Sensor Kinase KdpD Occurs via the Universal Stress Protein Domain and Downregulates the Expression of the Kdp Potassium Transporter. Journal of Bacteriology 198, 98-110 (2016).

127. Huynh, T. N. et al. Cyclic di-AMP targets the cystathionine beta-synthase domain of the osmolyte transporter OpuC. Molecular Microbiology (2016). doi: $10.1111 / \mathrm{mmi} .13456$

128. Schuster, C. F. et al. The second messenger c-di-AMP inhibits the osmolyte uptake system OpuC in Staphylococcus aureus. Science Signaling 9, ra81 (2016).

129. Zhang, L., Li, W. \& He, Z.-G. DarR, a TetR-like transcriptional factor, is a cyclic diAMP-responsive repressor in Mycobacterium smegmatis. Journal of Biological Chemistry 288, 3085-3096 (2013).

130. Nelson, J. W. et al. Riboswitches in eubacteria sense the second messenger c-diAMP. Nature Chemical Biology 9, 834-839 (2013).

131. Gundlach, J., Rath, H., Herzberg, C., Mäder, U. \& Stülke, J. Second Messenger Signaling in Bacillus subtilis: Accumulation of Cyclic di-AMP Inhibits Biofilm Formation. Frontiers in microbiology 7, 804 (2016).

132. Peng, X., Zhang, Y., Bai, G., Zhou, X. \& Wu, H. Cyclic di-AMP mediates biofilm formation. Molecular Microbiology 99, 945-959 (2016).

133. Mehne, F. M. P. et al. Control of the diadenylate cyclase CdaS in Bacillus subtilis: an autoinhibitory domain limits cyclic di-AMP production. Journal of Biological Chemistry 289, 21098-21107 (2014).

134. Dengler, V. et al. Mutation in the C-di-AMP cyclase dacA affects fitness and resistance of methicillin resistant Staphylococcus aureus. PLOS ONE 8, e73512 (2013).

135. Ablasser, A. et al. cGAS produces a 29-59-linked cyclic dinucleotide second messenger that activates STING. Nature 498, 380-384 (2013).

136. Kato, K., Ishii, R., Hirano, S., Ishitani, R. \& Nureki, O. Structural Basis for the Catalytic Mechanism of DncV, Bacterial Homolog of Cyclic GMP-AMP Synthase. Structure 23, 843-850 (2015).

137. Nelson, J. W. et al. Control of bacterial exoelectrogenesis by c-AMP-GMP. Proceedings of the National Academy of Sciences 112, 5389-5394 (2015).

138. Kellenberger, C. A. et al. GEMM-I riboswitches from Geobacter sense the bacterial second messenger cyclic AMP-GMP. Proceedings of the National Academy of Sciences 112, 5383-5388 (2015).

139. Sun, L., Wu, J., Du, F., Chen, X. \& Chen, Z. J. Cyclic GMP-AMP Synthase Is a Cytosolic DNA Sensor That Activates the Type I Interferon Pathway. Science 339, 786791 (2013).

140. Civril, F. et al. Structural mechanism of cytosolic DNA sensing by cGAS. Nature 498, 332-337 (2013).

141. Diner, E. J. et al. The innate immune DNA sensor cGAS produces a noncanonical cyclic dinucleotide that activates human STING. Cell Rep 3, 1355-1361 (2013).

142. Kranzusch, P. J. et al. Ancient Origin of cGAS-STING Reveals Mechanism of Universal 2',3' cGAMP Signaling. Molecular Cell 59, 891-903 (2015).

143. Karaolis, D. K. R. et al. Bacterial c-di-GMP is an immunostimulatory molecule. $J$. 
144. McWhirter, S. M. et al. A host type I interferon response is induced by cytosolic sensing of the bacterial second messenger cyclic-di-GMP. Journal of Experimental Medicine 206, 1899-1911 (2009).

145. Woodward, J. J., Iavarone, A. T. \& Portnoy, D. A. c-di-AMP secreted by intracellular Listeria monocytogenes activates a host type I interferon response. Science 328, 1703-1705 (2010).

146. Andrade, W. A. et al. Group B Streptococcus Degrades Cyclic-di-AMP to Modulate STING-Dependent Type I Interferon Production. Cell Host \& Microbe 20, 49-59 (2016). 
Figure 1

a

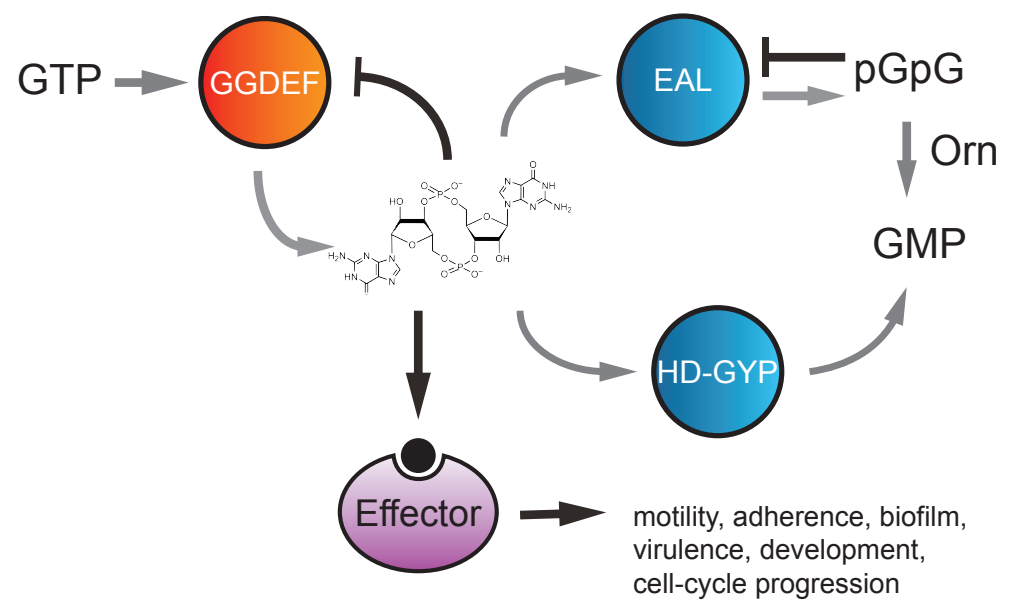

C

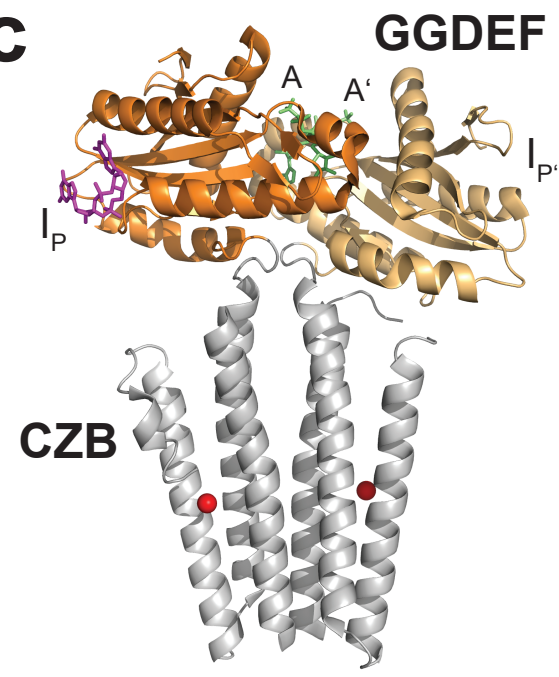

b

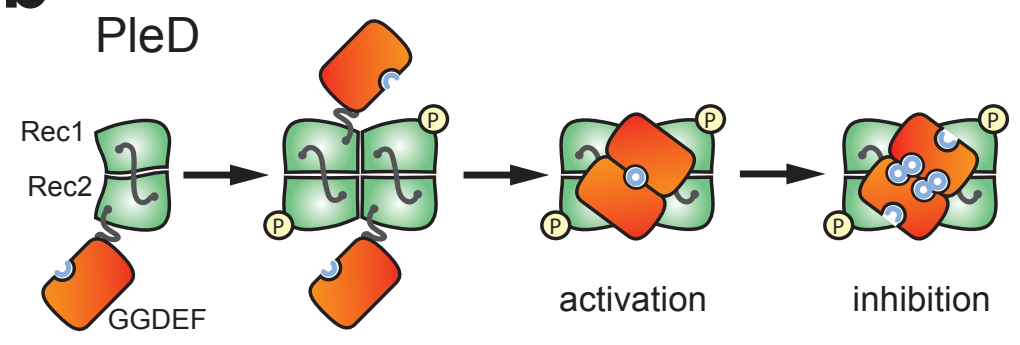

DgcZ

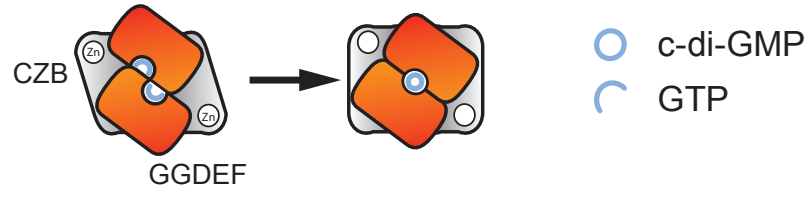

d

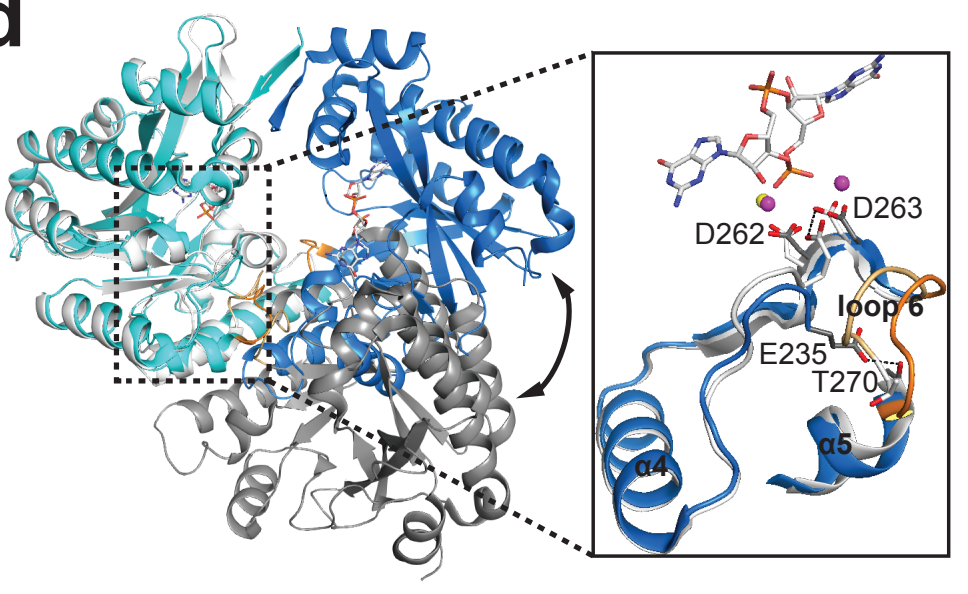

e
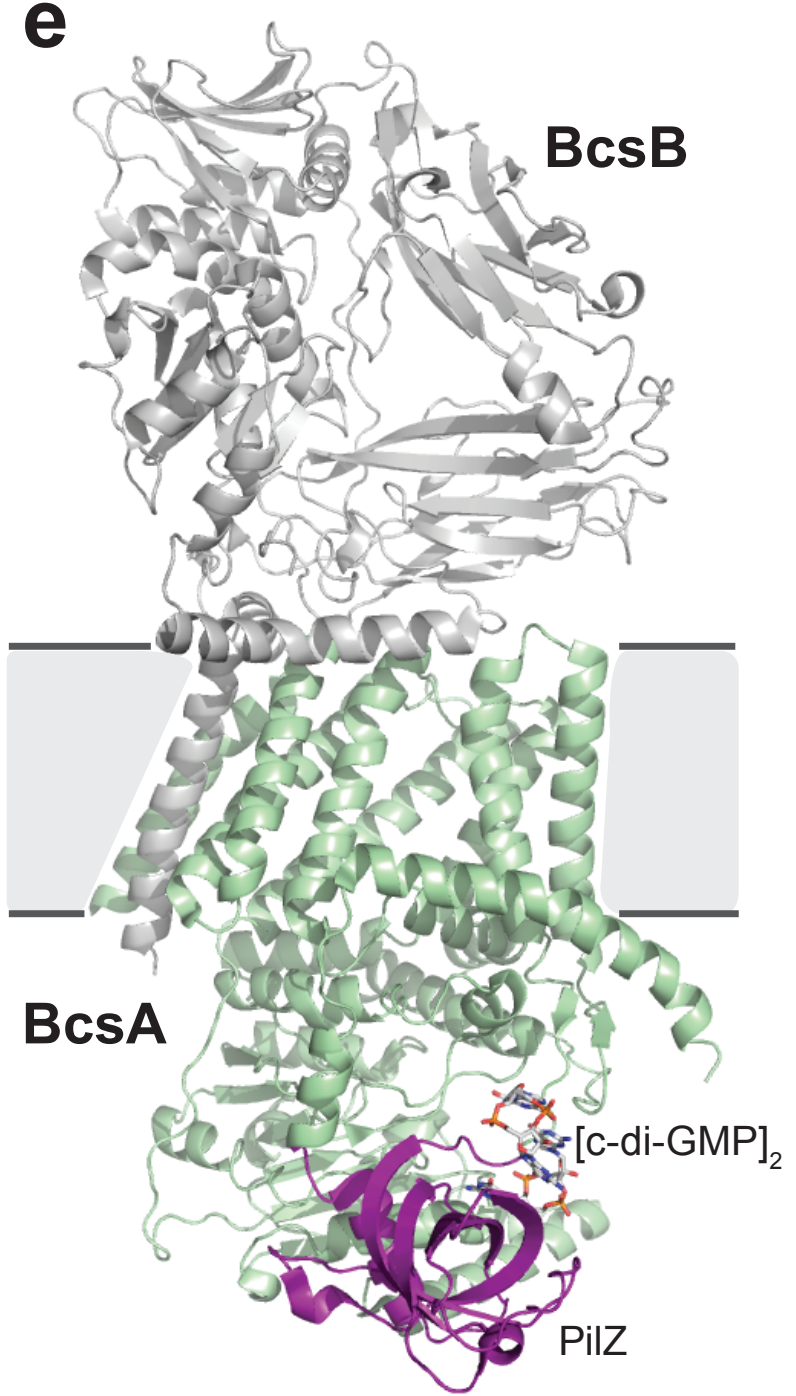
Figure 2

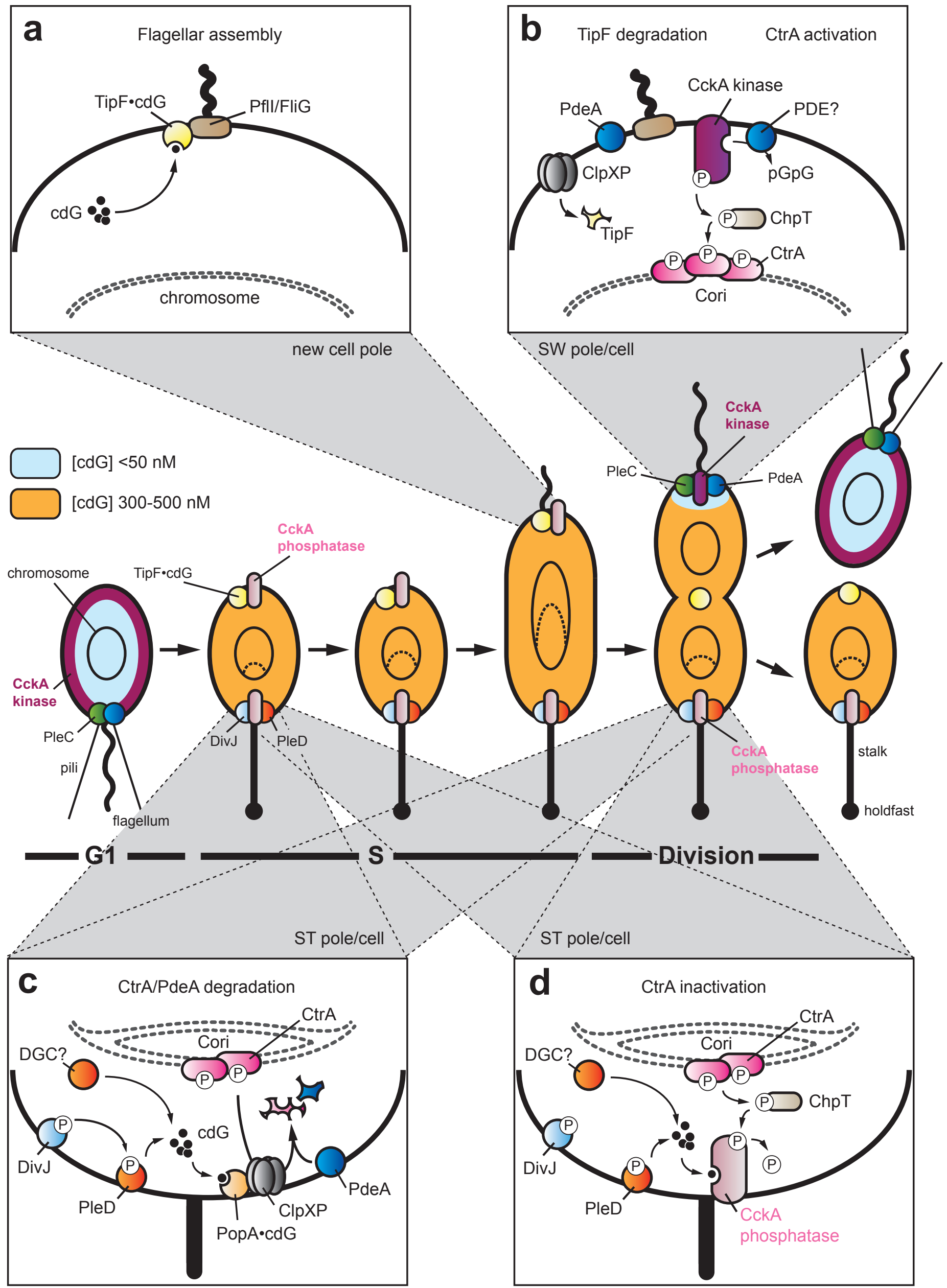




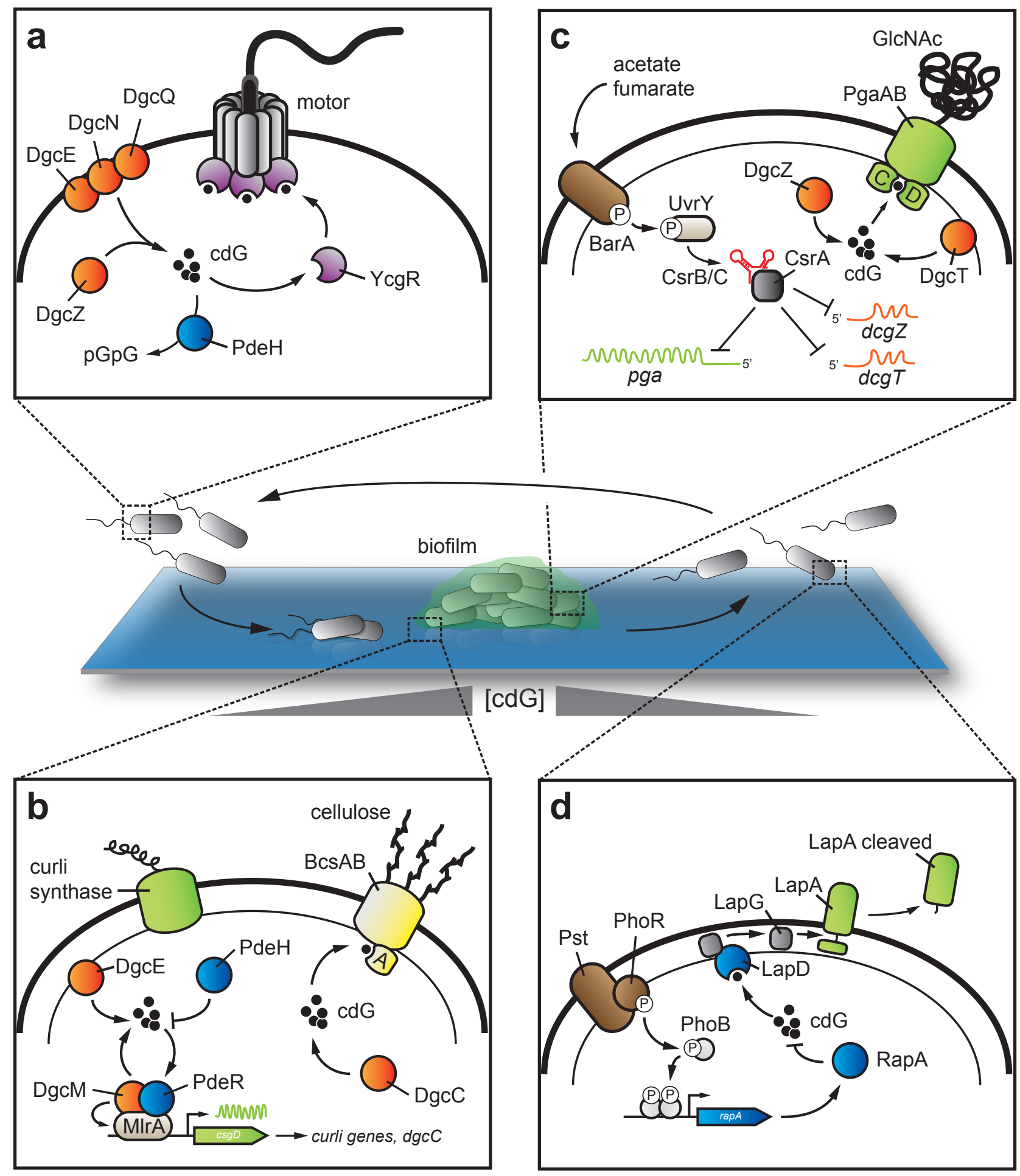


Figure 4

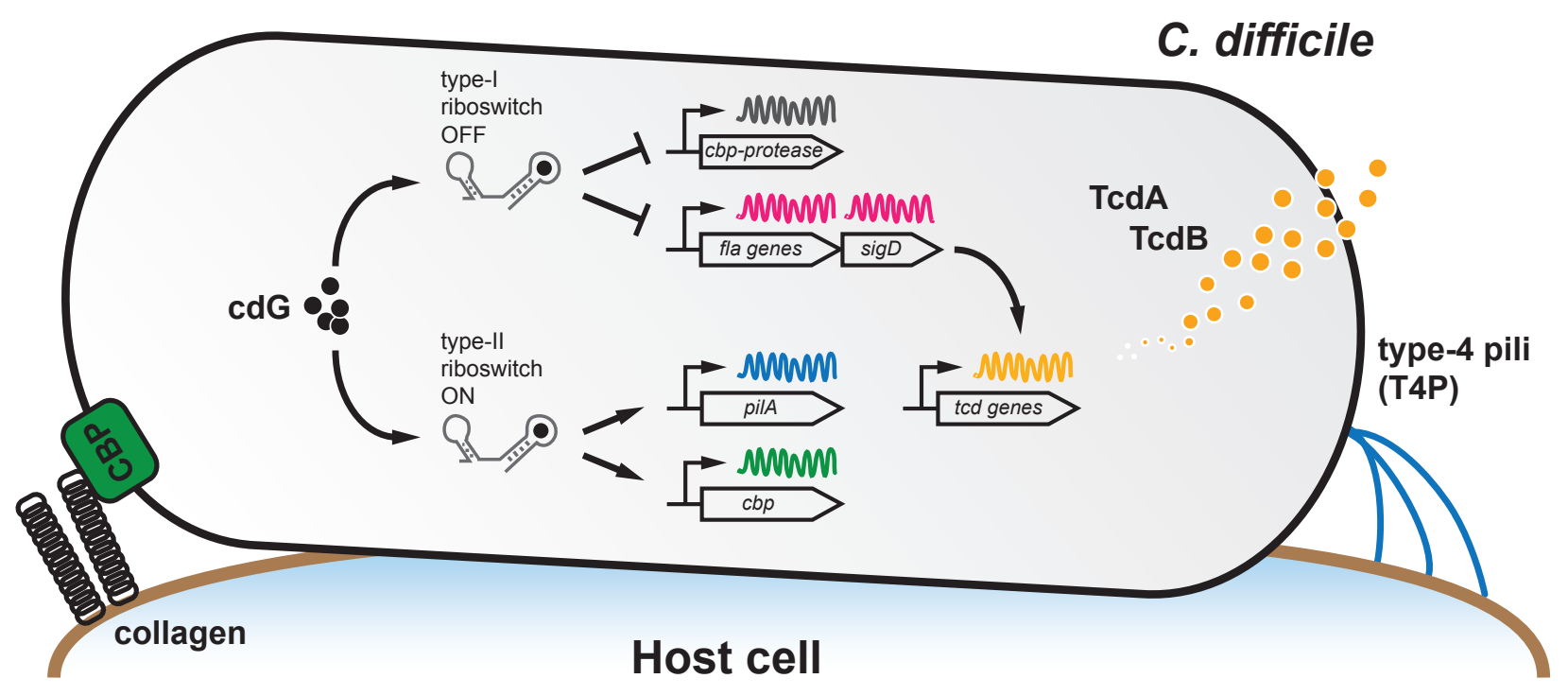


Figure 5
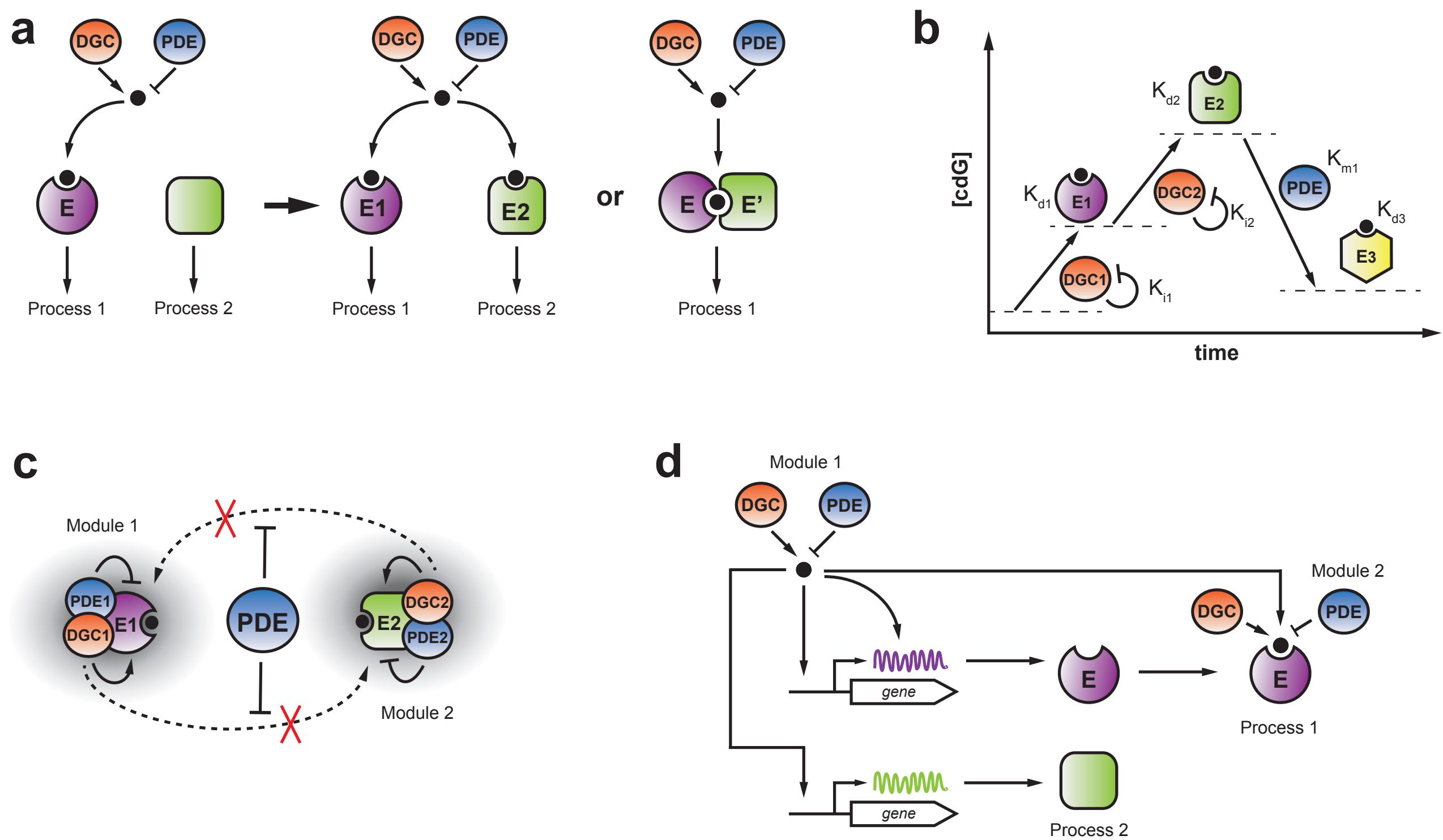